\title{
Elucidating the Role of Matrix Porosity and Rigidity in Glioblastoma Type IV Progression
}

\author{
Rui C. Pereira ${ }^{1}\left(\mathbb{D}\right.$, Raffaella Santagiuliana ${ }^{1,2}$, Luca Ceseracciu ${ }^{3}(0)$, Daniela P. Boso ${ }^{2}(\mathbb{D}$, \\ Bernhard A. Schrefler ${ }^{2,4}$ and Paolo Decuzzi 1,5,* \\ 1 Laboratory of Nanotechnology for Precision Medicine, Fondazione Istituto Italiano di Tecnologia, \\ Via Morego 30, 16163 Genoa, Italy; rui.pereira@iit.it (R.C.P.); sanatgiuliana.raffaella@gmail.com (R.S.) \\ 2 Dipartimento di Ingegneria Civile Edile e Ambientale, Università di Padova, Via Marzolo 9, \\ 35131 Padova, Italy; daniela.boso@unipd.it (D.P.B.); bernhard.schrefler@dicea.unipd.it (B.A.S.) \\ 3 Materials Characterization Facility, Fondazione Istituto Italiano di Tecnologia, Via Morego 30, \\ 16163 Genoa, Italy; luca.ceseracciu@iit.it \\ 4 Institute for Advanced Study, Technische Universität München, Lichtenbergstraße $2 \mathrm{a}$, \\ 85748 Garching bei München, Germany \\ 5 Department of Radiology, Stanford University, Palo Alto, CA 94305, USA \\ * Correspondence: paolo.decuzzi@iit.it; Tel.: +39-049-8275424; Fax: +39-049-8275604
}

Received: 13 November 2020; Accepted: 14 December 2020; Published: 18 December 2020

\begin{abstract}
The highly infiltrating nature of glioma cells is the major cause for the poor prognosis of brain malignancies. Motility, proliferation, and gene expression of cells in natural and synthetic gels have been analyzed by several authors, yet quantitative studies elucidating the role of matrix porosity and rigidity in the development of whole malignant masses are missing. Here, an experimental-computational framework is introduced to analyze the behavior of U87-MG cells and spheroids in compact hyaluronic acid gels (HA), replicating the brain parenchyma; and fibrous collagen gels (COL), resembling the organized structures of the brain. Experimentally it was observed that individual U87-MG cells in COL assumed an elongated morphology within a few hours post inclusion (p.i.) and travelled longer distances than in HA. As spheroids, U87-MG cells rapidly dispersed into COL resulting in infiltrating regions as large as tumor cores $(\approx 600 \mu \mathrm{m}$, at 8 days p.i.). Conversely, cells in HA originated smaller and denser infiltrating regions ( $\approx 300 \mu \mathrm{m}$, at 8 days p.i.). Notably, COL tumor core size was only $20 \%$ larger than in HA, at longer time points. Computationally, by introducing for the first time the effects of matrix heterogeneity in our numerical simulations, the results confirmed that matrix porosity and its spatial organization are key factors in priming the infiltrating potential of these malignant cells. The experimental-numerical synergy can be used to predict the behavior of neoplastic masses under diverse conditions and the efficacy of combination therapies simultaneously aiming at killing cancer cells and modulating the tumor microenvironment.
\end{abstract}

Keywords: cancer growth; matrix porosity; matrix rigidity and heterogeneity; multi-physics model

\section{Introduction}

Glioblastoma type IV (GBM) is the malignancy of the central nervous system with the poorest prognosis [1]. Standard treatments for glioblastoma type IV currently consist of surgical resection followed by radiotherapy and chemotherapy [2]. The median survival for GBM patients is nearly 14 months and the 5-year survival rate is only of 5\%. A distinctive feature of GBM cells is their ability to infiltrate the host tissue forming multiple micro-metastases which cannot be surgically removed or efficiently killed by molecular anticancer therapies. Spreading of GBM cells is believed to occur through two major pathways: across the extracellular matrix (ECM), which is mainly composed of a dense 
matrix of hyaluronic acid and proteoglycans; and along specific brain structures, called Scherer's structures, which include white matter tracts and blood vessels [3]. The migration mechanisms are pathway dependent and the ability of glioma cells to adopt different migratory strategies is key in priming the aggressiveness of this deadly disease.

Tumor cell motility has been extensively studied using classical 2D assays, including the monolayer wound-healing assay, the radial migration assay, and the Boyden chamber assay [4]. Although these systems can be efficiently used to assess the role of a variety of biochemical cues (adhesion proteins, chemoattractants, and so on) in modulating cell migration and proliferation, they cannot reproduce the complex set of biomechanical stimuli typically found in three-dimensional microenvironments [5-7]. The picture is further complicated by the fact that gel-like parenchyma coexist with ordered and more rigid structures within the brain tissue [8,9]. On the other hand, animal experiments and ex-vivo assays based on tissue slices, which would more closely recapitulate the biophysical features of a brain tumor microenvironment, are technically cumbersome, costly, and introduce additional variability which discourage any systematic analyses [10-12].

In this context, scaffolds made out of natural and artificial polymers offer a simple and reproducible solution for studying tumor cells in realistic microenvironments. Collagen (COL), poly(ethylene glycol) (PEG), hyaluronic acid (HA), chitosan, and combinations thereof, are among the most extensively used polymers for these applications [13-19]. For instance, COL and PEG-based hydrogels were used to demonstrate that HA inclusions tend to inhibit migration and favor aggregation of brain tumor cells [14-16]. Similar hydrogels were also employed to demonstrate that matrix porosity supports the rapid migration of GBM cells and that ECM remodeling favors cell tumor spreading more than proliferation [17-19]. In particular Yang et al. [17] suggested within the framework of biochemistry of glioma invasion in different collagen gels that pore size is a key parameter determinant of glioma invasive speed in these gels, independent of global collagen concentration and both local and bulk stiffness. On the other hand, Koike et al. [20] have shown that Hyaluronan can support formation of cancer cell aggregates. Koike et al. [20] deal with Dunning R3327 rat prostate carcinoma AT3.1 cells, which predominantly do not grow as spheroids in free suspension. However, it is found that solid stress facilitates the formation of spheroids and that relieving solid stress by enzymatic digestion of gels resulted in gradual loss of spheroidal morphology in 8 days. In contrast, the low metastatic variant AT2.1 cells, which grow as spheroids in free suspension as well as in the gels, maintained their spheroidal morphology even after stress removal. Koike et al. [20] suggest that cell-cell adhesion may be adequate for spheroid formation, whereas solid stress may be required to form spheroids when cell-matrix adhesion is predominant. In the case of the glioblastoma cells however, Santagiuliana et al. [21] have shown that these cells grow as spheroids in free suspension. The cell-to matrix adhesion has also been investigated and it has been found that lower values of adhesion result in larger radii of the spheroid. Further in Mascheroni et al. [22,23] the growth of U-87MG spheroids has been investigated both freely growing in the culture medium and subjected to an external mechanical pressure induced by a Dextran solution showing both experimentally and numerically that increasing confinement stress simply reduces the radius of the spheroids. Finally Hatzikirou et al. [24] propose that in glioblastoma the transition to invasive tumor phenotypes can be explained on the basis of the microscopic 'Go or Grow' mechanism i.e., the mutually exclusive migration/proliferation dichotomy [25]. The driving force in this case is not mutation but oxygen shortage, i.e., hypoxia in the environment of a growing tumor. This hypothesis was tested with the help of a lattice-gas cellular automaton (LGCA). This interesting hypothesis, although possible to simulate with our growth model is not pursued here since, in light of the previous work, we concentrate in the following on matrix porosity and morphology.

Our domain of investigation is within the framework of transport oncophysics [26,27], where it is assumed that physical properties of biological barriers control cell, particle, and molecule transport across tissues as well as invasion and infiltration. This transport and its deregulation play an overarching role in cancer physics. The concept of biological barriers and their effect on transport has brought a new understanding of how transport can modulate cancer biology and therapeutic efficacy. We show that 
based on purely physical reasoning we can explain and model the experimentally observed behavior of U-87MG cells and aggregates.

To this aim 3D scaffolds are realized using hyaluronic acid hydrogels (HA), mimicking the dense and gel-like brain parenchyma, and collagen hydrogels (COL), resembling, at first approximation, the ordered structures of the brain. Then, morphology and migration patterns of individual malignant cells of human glioblastoma type IV cells (U87-MG) are monitored via fluorescent and electron microscopies. The complex growth behavior of spheroids encapsulated in HA and COL are also documented over time, discriminating between the dense tumor core and the surrounding infiltrating region. Finally, a multiphase computational model is employed to predict tumor growth and quantitatively elucidate the role of matrix morphology and porosity on the macroscopic behavior of GBM cells.

\section{Materials and Methods}

\subsection{Materials}

Collagen type I from bovine origin, agarose type II, and bromuro di 3-(4,5-dimetiltiazol-2-il)2,5-difeniltetrazolio (MTT) were acquired from Sigma Aldrich (St. Louis, MO, USA). Hyaluronic acid Corgel ${ }^{\mathrm{TM}}$ BioHydrogel (TSNaHy, $263.3 \mathrm{~g} / \mathrm{mol}$ ) was purchased from Lifecore Biomedical (Lifecore Biomedical, LLC, Chaska, MN, USA). Glass slides and coverslips were acquired from Fisher Scientific (Philadelphia, PA, USA). Human glioblastoma type IV cell line U87-MG (ATCC ${ }^{\circledR}$ HTB-$^{-14^{\mathrm{TM}}}$ ) and Eagle's minimum essential medium (EMEM) were purchased from ATCC@ (ATCC $\subset$, Manassas, VA, USA). Phosphate buffered saline (PBS), EMEM powder (Gibco, Monza, Italy) and fetal calf serum (FCS, Invitrogen, Monza, Italy) were purchased from Life Technologies ${ }^{\mathrm{TM}}$ (Eugene, OR, USA). The 96 multiwell GravityTRAPTM ULA plates were acquired from PerkinElmer (PerkinElmer, Waltham, MA, USA).

\subsection{Cell Culture and Spheroid Formation}

Human glioblastoma U87-MG cells (American Type Culture Collection, ATCC) were selected for their high invasiveness and used as a preclinical model of glioblastoma type IV. Cells were cultured in standard Essential Eagle's Minimum Essential Medium (EMEM) supplemented with 10\% fetal bovine serum (FBS) and 1\% antibiotics (Euroclone). Medium was changed every 2 days and cells in all experiments were used at passage 8, or lower. U87-MG GFP ${ }^{+}$were kindly donated by Dr. Davide De Petri Tonelli at the Italian Institute of technology in Genova. U87-MG GFP ${ }^{+}$spheroids were generated using nonadhesive GravityTRAP ${ }^{\mathrm{TM}} 96$ multiwell plates according to the manufacturer's protocol. In brief, cells were washed twice with PBS, detached by trypsin and counted. Then, 500 cells in $60 \mu \mathrm{L}$ were dropped inside each well of the 96 multiwell plates. Multiwell plates were centrifuged at 250 RCF (relative centrifugal force) for $2 \mathrm{~min}$ at room temperature. Spheroids were kept in culture for $24 \mathrm{~h}$ prior encapsulation into hydrogels.

\subsection{Preparation of Hydrogels and Cell Encapsulation}

Collagen type I solution $(3 \mathrm{mg} / \mathrm{mL})$ was mixed with a ratio of 4:1 $(v / v)$ with a phosphate buffered saline solution with EMEM (5×) with phenol red, $0.1 \mathrm{M} \mathrm{NaOH}$, and DNA/RNA free sterile water adapted to cell culture to yield a collagen concentration of $2 \mathrm{mg} / \mathrm{mL}$ at $\mathrm{pH}$ of 7.4. Corgel ${ }^{\mathrm{TM}}$ hyaluronic acid hydrogel was prepared according to supplier instructions (Lifecore Biomedical LLC, Chaska, MN, USA) with minor adjustments [28]. Briefly, polymer was dissolved in horseradish peroxidase in PBS (10 U/mL) solution provided by the supplier to obtain a solution of $5 \mathrm{mg} / \mathrm{mL}$. Afterwards, $50 \mu \mathrm{L}$ of polymer solution was mixed with cells resuspended in $60 \mu \mathrm{L}$ of EMEM completed medium. Crosslinking reaction was initiated by addition of hydrogen peroxide $\left(\mathrm{H}_{2} \mathrm{O}_{2}\right)$ with a final ratio of 1:25 $(v / v)$. In both hydrogel preparations, U87-MG GFP ${ }^{+}$were encapsulated at a final concentration of $2 \times 10^{6} \mathrm{cells} / \mathrm{mL}$, as a single cell suspension. Volume calculations were performed to achieve the 
above-mentioned cellular concentration on both gels. In the case of spheroids, one individual spheroid was encapsulated within each single hydrogel. To this aim, spheroids were added individually to the different hydrogels (COL and HA) in a volume of $60 \mu \mathrm{L}$ each. Hydrogels were kept in culture and medium was changed every 2 days.

\subsection{Mechanical Characterization of Hydrogels}

Collagen type I and hyaluronic acid hydrogels were characterized mechanically via confined compression tests on a dual column universal testing machine (Instron 3365). Both hydrogels were deposited on 12-well culture plates (diameter $22 \mathrm{~mm}$ ) and tested using a $20 \mathrm{~mm}$ diameter stainless steel piston with a loading rate of $1 \mathrm{~mm} / \mathrm{min}$. This allowed the liquid to permeate through the gap between the well and piston. The aggregate modulus $H_{a}$ was extracted from the initial slope of the stress-strain curves, corresponding to a deformation smaller than $5 \%$. Then the aggregate modulus was used to calculate the Young's modulus $E$ according to the equation $H_{a}=\frac{E(1-v)}{(1+v)(1-2 v)}$, where the Poisson ratio was assumed to be $v=0.45$.

\subsection{Scanning Electron Microscopy (SEM)}

At different time points, hydrogels were collected for SEM analysis. In brief, samples were carefully washed twice with PBS and afterwards fixed in a solution of $2 \%$ Glutaraldehyde and $2 \%$ Paraformaldehyde in Na-Cacodylate buffer $0.1 \mathrm{M}$ for $2 \mathrm{~h}$ at room temperature. Then, hydrogels were immersed in Na-Cacodylate $0.1 \mathrm{M}$ buffer solution with $1 \% \mathrm{OsO}_{4}$ and $1.5 \%$ Hexayanoferrate for $2 \mathrm{~h}$, at room temperature. Samples were then washed in distillated water and dehydrated by serial immersions in ethanol solutions (30\%, 50\%,70\%,90\%, 96\%, and 100\%), for $10 \mathrm{~min}$ each. Then, hydrogels were immersed in sequential series of ethanol/bis (trimethylsilyl) amine (HMDS) mixtures (3:1; 1:1;1:3) for $20 \mathrm{~min}$ each. Samples were kept overnight in pure HMDS solution until total evaporation of the solvent. Afterwards, samples were sputtered with $10 \mathrm{~nm}$ of gold and images were acquired with JEOL JSM 6490-LA (JEOL, Tokyo, Japan) microscope with accelerating voltage of $10 \mathrm{kV}$.

\subsection{Optical Microscopy Analyses}

Spatial distribution and morphology of encapsulated U87-MG GFP ${ }^{+}$cells within collagen type I and hyaluronic acid hydrogels was accessed by the use of a fluorescent inverted microscope (Leica DMI6000 B, Milano, Italy) equipped with a high-speed camera (Leica DFC360 FX, Milano, Italy). Images were acquired by using bright field and $488 \mathrm{~nm}$ filters. Spheroid growth, inside the hydrogels, was followed by the use of the same optical microscope. Images were acquired over time using the above cited filters. Tumor size was calculated by measuring the spheroid perimeter via ImageJ64 tools (https://imagej.nih.gov/ij/).

\subsection{Time Lapse Acquisition and Cell Trajectory Analyses}

Cell behavior within collagen type I and hyaluronic acid hydrogels was monitored using an automated all-in-one microscope chamber (Eclipse TiE, Nikon, and Okolab), with controlled temperature and humidity. Prior to time-lapse acquisition, U87-MG GFP ${ }^{+}$cells were encapsulated within both different hydrogels, as described previously. Hydrogels were prepared inside 48 multiwell-plates precoated with agarose to avoid cell adhesion at the bottom of the wells. Cells were encapsulated at a density of $5 \times 10^{5}$ cells/mL in both hydrogels (lower than the initial cell concentration) to allow better cell tracing. Hydrogels were cultured in EMEM supplemented with $10 \%$ FBS. Phase-contrast and fluorescent images were captured every $10 \mathrm{~min}$ for $24 \mathrm{~h}$. Moving trajectories of 50 cells on each hydrogel (five different hydrogels for studied condition) were analyzed. 


\subsection{MTT Assay}

The proliferation of encapsulated U87-MG cells within both gels was evaluated at different time points, using thiazolyl blue staining (MTT, Sigma-Aldrich, St. Louis, MO, USA), in which absorbance data correlate with cell metabolic activity. In brief, at each time point, the medium was removed and replaced with $1 \mathrm{~mL}$ fresh medium supplemented with MTT stock solution at a final concentration of $5 \mathrm{mg} / \mathrm{mL}$. After $3 \mathrm{~h}$ incubation, the medium was removed, and the converted dye was solubilized by the addition of $1 \mathrm{~mL}$ ethanol absolute. To obtain a homogenous solution, hydrogels were disrupted mechanically via a potter [29]. Absorbance was measured at $570 \mathrm{~nm}$ with background subtraction at $670 \mathrm{~nm}$.

\subsection{Cellular Metrics: Single Cell Mobility and Spheroid Progression Kinetics}

NIS software from Nikon was used to quantify single cell mobility inside both hydrogels. Tumor spheroid metastatic progression inside collagen type I and hyaluronic acid hydrogels was analyzed via ImageJ64 tools (https://imagej.nih.gov/ij/). At each time point, the region occupied by the tumor was separated into two domains- the Tumor Core and the Tumor Infiltrating Region. The size of both domains was measured over time.

\subsection{Statistics}

Values of U87-MG proliferation inside collagen type I and hyaluronic acid hydrogels obtained over time are expressed as a mean \pm standard deviation, with $n=3$ for each group of culture. Spheroid metastasis kinetic values are expressed as a mean \pm standard deviation with $n=3$, for each group of culture from three independent assays. Statistical significance was determined using Student's $t$-test multiple comparison procedure at a confidence level of $95 \%{ }^{* *}(p<0.005),{ }^{*}(p<0.05)$, via GraphPad (www.Graphpad.com).

The coefficient of determination $R^{2}$ is used to quantify the accuracy of the computational model against the experimental data. It is defined as $R^{2}=\sum_{i}\left(\hat{y}_{i}-\bar{y}\right)^{2} / \sum_{i}\left(y_{i}-\bar{y}\right)^{2}$, where $y_{i}$ are the experimental data, $\bar{y}$ is the average value of experimental data, $\hat{y}_{i}$ are the results predicted with the model. A $R^{2}$ of 1 indicates perfect agreement between computational predictions and experimental data.

\section{Computational Model}

Our tumor growth model $[30,31]$ is a continuum model of the multiphysics type and includes aspects of Thermodynamically Constrained Averaging Theory [32] where the model derivation proceeds systematically from known microscale relations to mathematically and physically consistent larger scale relations. Other continuum growth models are of the multiparameter type [33-35] based on mixture theory where the relevant balance equations are written directly at the level of interest and the thermodynamic consistency is satisfied at the same level. Our model comprises four phases: the solid phase, i.e., the ECM and three fluid phases: tumor cells, TCs, host cells, HCs, interstitial fluid, IF. In this paper, to simulate the growth of U87-MG cells cultured in collagen type I hydrogels and in HA hydrogels, the system is reduced to three phases, ECM, TCs, IF, by taking the volume fraction of HCs, $\varepsilon^{h}$, equal to zero.

ECM is represented by an elasto-visco-plastic porous solid, with porosity $\varepsilon$, filled by TCs and IF. It is recalled that pore size as used in Yang et al. (2010) is not a direct measure of porosity. Both can be measured in biological tissues via scanning electron microscopy (SEM) and microcomputed tomography and from these a relation between the two can be established. In Karageorgiou and Kaplan [36], there are some values of both pore size and porosity for biological tissues such as collagen gels and hyaluronic acid gels. Our chosen values of the porosity for modeling purposes are consistent with those values.

At each point of the continuum, the representative elementary volume (REV) of the multiphase computational model comprises a certain portion of ECM, TCs, and IF, as schematically shown in 
Figure 1. The TCs are divided in living tumor cells, LTCs, and necrotic tumor cells, NTCs. In the IF phase, transported species (nutrients) are considered. The volume fraction of the solid phase ECM is $\varepsilon^{s}=1-\varepsilon$. The other phases, tumor cells $\left(\varepsilon^{t}\right)$ and interstitial fluid $\left(\varepsilon^{l}\right)$, occupy the rest of the volume so that the volume fractions for all phases add up to unity.

$$
\varepsilon^{s}+\varepsilon^{t}+\varepsilon^{l}=1
$$

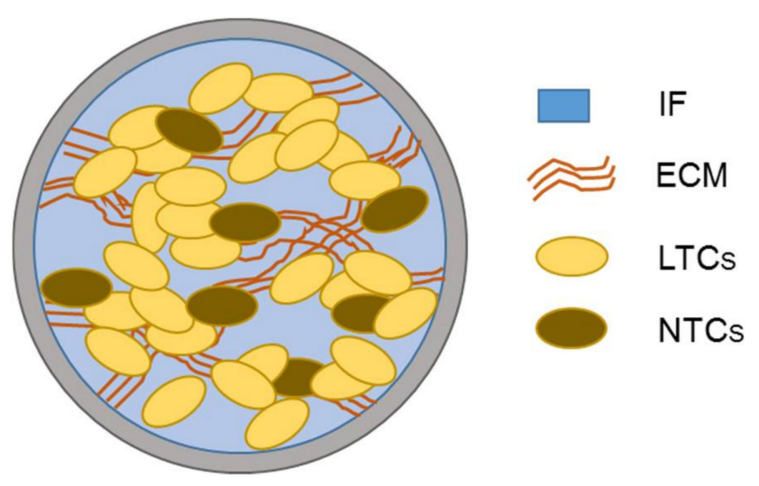

Figure 1. Representative elementary volume. Schematic representation of the representative elementary volume (REV) used in the simulations. This comprises the three phases of the problem: tumor cells (TCs), divided in living and necrotic ones, interstitial fluid (IF), and the extracellular matrix (ECM).

\subsection{Governing Equations}

The governing equations of the model used here are the mass balance equation for ECM, IF, TCs, nutrients, and the linear momentum balance equation for the mixture ECM+IF+TCs. The following equations are obtained from the general forms by introducing some simplifications and closure relationships (e.g., a Fickian type equation for diffusion of species, a generalized Darcy's equation for flow of the fluid phases, etc.). The primary variables of the model are differential pressures between phases $t, h, l, p^{h l}$ and $p^{t h}$, IF pressure $p^{l}$, nutrient mass fraction, and displacement $u^{s}$ of solid phase (ECM). It is recalled that $p^{t h}+p^{h l}=p^{t l}$.

The saturation degree of a fluid phase $\alpha$ is $S^{\alpha}=\varepsilon^{\alpha} / \varepsilon$, with $\alpha=t, h, l$ for TCs, HCs, and IF, respectively. In this paper the saturation degree of HCs is $S^{h}=0$. From Equation (1) the constraint condition for saturation degrees for the system with just three phases follows

$$
S^{t}+S^{l}=1
$$

The mass balance equation of the ECM is

$$
\frac{\partial \varepsilon}{\partial t}=\nabla \cdot \mathbf{v}^{\bar{s}}+\frac{(1-\varepsilon)}{\rho^{s}} \frac{\partial \rho^{s}}{\partial t}-\nabla \cdot\left(\varepsilon \mathbf{v}^{\bar{s}}\right)-\frac{\begin{array}{c}
l \rightarrow s \\
M \\
E C M
\end{array}}{\rho^{s}}
$$

The mass balance equation of TCs reads

$$
\begin{aligned}
& {\left[\frac{\varepsilon S^{t}}{K_{t}}+\frac{S^{t}(1-\varepsilon)}{K_{S}}\left(S^{t}+p^{t h} \frac{\partial S^{t}}{\partial p^{t h}}\right)+\varepsilon \frac{\partial S^{t}}{\partial p^{t h}}\right] \frac{\partial p^{t h}}{\partial t}+\left[\frac{\varepsilon S^{t}}{K_{t}}+\frac{S^{t}(1-\varepsilon)}{K_{S}}\left(1-S^{l}-p^{h l} \frac{\partial S^{l}}{\partial p^{h l}}\right)\right] \frac{\partial p^{h l}}{\partial t}+} \\
& +\left[\frac{\varepsilon S^{t}}{K_{t}}+\frac{S^{t}(1-\varepsilon)}{K_{s}}\right] \frac{\partial p^{l}}{\partial t}=\nabla \cdot\left[\frac{k_{r e l}^{t} \mathbf{k}}{\mu^{t}} \cdot \nabla\left(p^{l}+p^{h l}+p^{t h}\right)\right]-S^{t}\left(\mathbf{1}: \mathbf{d}^{\overline{\bar{s}}}\right)-\nabla S^{t} \cdot\left(\varepsilon \mathbf{v}^{\bar{s}}\right)+ \\
& +\frac{1}{\rho^{t}}(\underset{\text { growth }}{\stackrel{l \rightarrow t}{M}}-\stackrel{t \rightarrow l}{M})+S_{\text {lysis }}^{t} \frac{\stackrel{l \rightarrow s}{M}}{\rho^{s}}
\end{aligned}
$$


The mass balance equation of IF reads

$$
\begin{aligned}
& \frac{S^{l}(1-\varepsilon)}{K_{S}}\left(p^{t h} \frac{\partial S^{t}}{\partial p^{t h}}+S^{t}\right) \frac{\partial p^{t h}}{\partial t}+\left[\frac{S^{l}(1-\varepsilon)}{K_{s}}\left(1-p^{h l} \frac{\partial S^{l}}{\partial p^{h l}}-S^{l}\right)+\varepsilon \frac{\partial S^{l}}{\partial p^{h l}}\right] \frac{\partial p^{h l}}{\partial t}+ \\
& +\left(\frac{S^{l}(1-\varepsilon)}{K_{s}}+\frac{\varepsilon S^{l}}{K_{l}}\right) \frac{\partial p^{l}}{\partial t}=\nabla \cdot\left[\frac{k_{r e l}^{l} \mathbf{k}}{\mu^{l}} \cdot \nabla p^{l}\right]-S^{l}\left(\mathbf{1}: \mathbf{d}^{\overline{\bar{s}}}\right)-\nabla S^{l} \cdot\left(\varepsilon \mathbf{v}^{\bar{s}}\right)-\frac{1}{\rho^{l}}(\underset{\text { growth }}{\stackrel{l \rightarrow t}{M}-\underset{l y s i s}{M}})-\frac{t \rightarrow l}{\rho^{l}} \stackrel{i \rightarrow s}{M}
\end{aligned}
$$

where $\mu^{\alpha}$ is the dynamic viscosity, $k_{r e l}^{\alpha}$ is relative permeability, $\rho^{\alpha}$ is the density. Additionally, $\mathbf{d}^{\overline{\bar{s}}}$ is the Eulerian rate of strain tensor, $\mathbf{k}$ the intrinsic permeability tensor of the ECM, and $\underset{\text { growth }}{\stackrel{l}{M}}$ is an interphase exchange of mass between the phases $l$ and $t$, and represents the mass of IF consumed by tumor cell growth. $K_{i}$ is the compressibility of phase $i$ (with $i=s, t, h$ and $l$ ). $\stackrel{t \rightarrow l}{M}$ lysis takes into account of mass exchange between the necrotic compartment of tumor cells and the IF phase and $\underset{E C M}{M}$ takes account of ECM deposition by the living tumor cells.

The mass balance equation of the nutrient reads

$$
\varepsilon S^{l} \frac{\partial \omega^{n l}}{\partial t}-\nabla \cdot\left(\varepsilon S^{l} D_{e f f}^{n l} \nabla \omega^{n l}\right)=\frac{1}{\rho^{l}}\left(\omega^{n l}\left(\left(\begin{array}{c}
\stackrel{l \rightarrow t}{M} \\
\text { growth }
\end{array}-\underset{l y s i s}{M}\right)+\underset{E C M}{M}\right)-\stackrel{n}{M}^{M}\right)-\varepsilon S^{l} \mathbf{v}^{\bar{l}} \nabla \omega^{n l}
$$

where $D_{e f f}^{\overline{n l}}$ is the effective diffusivity of the nutrient species in the extracellular space, $\omega^{n l}$ the mass fraction of nutrient species $n$, and $\stackrel{n l}{M}$ t is the mass of nutrient consumed by tumor cells via metabolism and growth. In our simulations the oxygen is the sole nutrient considered.

A further governing equation of the model is the linear momentum balance of the mixture expressed in rate form as

$$
\nabla \cdot\left(\frac{\partial \mathbf{t}_{e f f}^{\overline{\bar{s}}}}{\partial t}-\bar{\alpha} \frac{\partial p^{s}}{\partial t} \mathbf{1}\right)=0
$$

where the interaction between the solid and the three fluid phases is accounted for through the effective stress $\mathbf{t}_{\text {eff }}^{\overline{\bar{s}}}$ in the sense of porous media mechanics

$$
\mathbf{t}_{e f f}^{\overline{\bar{s}}}=\mathfrak{t}^{\overline{\bar{s}}}+\bar{\alpha} p^{s} \mathbf{1}
$$

where $\mathbf{1}$ is the unit tensor, $\mathbf{t}^{\overline{\bar{s}}}$ is the total stress tensor in the solid phase, $\bar{\alpha}$ is the Biot's coefficient $\bar{\alpha}=1-K / K_{s}$, with $K$ the compressibility of the empty ECM. In the modeled problem, $K / K_{s}$ tends to zero hence we can assume a Biot's coefficient equal to 1 . Being $S^{h}=0$ the solid pressure $p^{s}$ is given as,

$$
p^{s}=p^{l}+\left(1-S^{l}\right) p^{h l}+S^{t} p^{t h}=p^{l}+S^{t} p^{t l}
$$

For the fluid phases the momentum balance equations can be simplified to

$$
\varepsilon^{\alpha} \nabla p^{\alpha}+\mathbf{R}^{\alpha} \cdot\left(\mathbf{v}^{\bar{\alpha}}-\mathbf{v}^{\bar{s}}\right)=0
$$

where $\mathbf{R}^{\alpha}$ is the resistance tensor that accounts for the frictional interactions between phases when there is flow and different phases have different velocities. With the generalized Darcy law, the resistance tensor is

$$
\left(\mathbf{R}^{\alpha}\right)^{-1}=\frac{k_{r e l}^{\alpha} \mathbf{k}}{\mu^{\alpha}\left(\varepsilon^{\alpha}\right)^{2}} \quad(\alpha=h, t, l)
$$

with $\mu^{\alpha}$ the dynamic viscosity that is defined to model the adhesion between tumor cells and ECM. 
The constitutive relationships for the fluid phases and the solid phase as well as the constitutive equations for growth and nutrient consumption have been extensively dealt with in $[30,31,37]$. Diffusion of the chemical species dissolved in the interstitial fluid follows Fick's law [30,31]. The constitutive relationship for the deformable solid phase (ECM) follows an elasto-viscoplastic law of Perzyna type in large deformation regime. Until a yield limit of the effective stress is reached, the solid behavior is contained in the elastic range and then it becomes visco-plastic with a constitutive flow rule obtained from rate-independent hardening plasticity by the introduction of the viscosity. For the numerical implementation of the equations see Appendix A.

\subsection{Description of Geometry, Boundary, and Initial Conditions of the Simulations}

The geometry of the problems at hand is simulated with a sphere segment in axisymmetric conditions with radius of $2500 \mu \mathrm{m}$. At the initial time instant, the multicellular tumor spheroid (MTS) is composed of three phases: (i) the TCs phase, in the red area of Figure 2 with radius of $200 \mu \mathrm{m}$ for the simulation of tumor growing in collagen and $250 \mu \mathrm{m}$ for the simulation of tumor growing in hyaluronic acid; (ii) the IF phase which fills the whole domain; (iii) the ECM solid phase which also fills the whole domain. The atmospheric pressure is taken as the reference pressure and the initial IF pressure is zero $\mathrm{Pa}$ in the entire domain. The initial volume fraction of TCs is 0.02 , the HCs are not present.

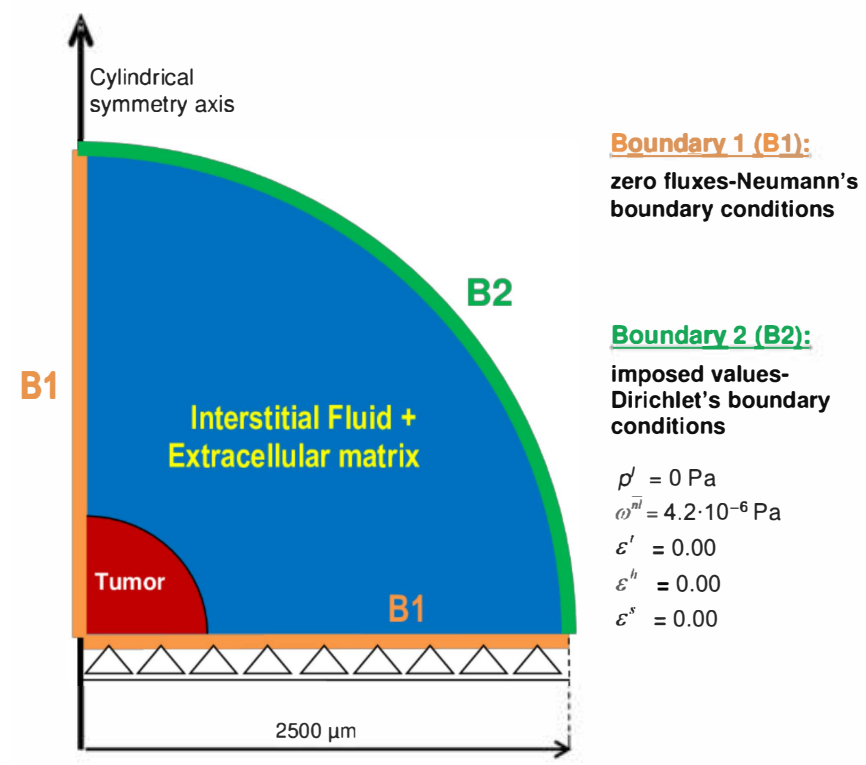

Figure 2. Geometry and boundary conditions of the problem. The computational domain is modeled as a spherical sector under axisymmetric conditions with a total radius of $2500 \mu \mathrm{m}$. Initially, the tumor spheroid is composed of a TCs phase (red region, with a radius of about $200 \mu \mathrm{m}$ for tumor growing in Collagen type I (COL) and $250 \mu \mathrm{m}$ for tumor growing in Hyaluronic Acid (HA), the IF phase, and ECM which fill the whole domain. The atmospheric pressure is taken as the reference pressure and the initial IF pressure is zero in the entire domain. The initial volume fraction of TCs is 0.02 .

Boundary conditions are imposed as indicated in Figure 2. To allow IF flux at the outer boundary the IF pressure is fixed there to zero Pa. Due to the symmetry of the problem there is no flux normal to the radius of the sphere segment. Oxygen is the sole nutrient species and its mass fraction is fixed to $\omega_{\text {env }}^{\overline{n l}}=4.2 \cdot 10^{-6}$ at the outer boundary and throughout the computational domain at initial time.

All model parameters are listed in Tables 1-3 classified by type. Coefficients $\gamma_{\text {growth }}^{\bar{n} l}$ and $\gamma_{0}^{\overline{n l}}$ control the TCs uptake of oxygen; they allow modeling consumption due to TCs growth and their metabolism. 
Table 1. Parameters depending on cells' type and IF.

\begin{tabular}{cccc}
\hline Parameter & Symbol & Value & Unit \\
\hline Density of the fluid phases $(\alpha=t$ and $l)$ & $\rho^{\alpha}$ & 1000 & $\mathrm{~kg} / \mathrm{m}^{3}$ \\
Dynamic viscosity of IF & $\mu^{l}$ & $1 \times 10^{-2}$ & $\mathrm{~Pa} \cdot \mathrm{s}$ \\
Dynamic viscosity of TCs & $\mu_{0}^{t}$ & 20 & $\mathrm{~Pa} \cdot \mathrm{s}$ \\
Adhesion of TCs & $\psi^{t}$ & 0 & $\mathrm{~Pa} / \mathrm{m}$ \\
Critical mass fraction of oxygen & $\omega_{\text {crit }}^{\overline{n l}}$ & $1.0 \times 10^{-6}$ & - \\
Growth coefficient of tumor cells [31] & $\gamma_{\text {growth }}^{t}$ & $2.0 \times 10^{-2}$ & $\mathrm{~kg} /\left(\mathrm{m}^{3} \cdot \mathrm{s}\right)$ \\
Necrosis coefficient [31] & $\gamma_{\text {necrosis }}^{t}$ & $1.0 \times 10^{-2}$ & $\mathrm{~kg} /\left(\mathrm{m}^{3} \cdot \mathrm{s}\right)$ \\
Consumption related to growth [31] & $\gamma_{\text {growth }}^{n l}$ & $1 \times 10^{-4}$ & $\mathrm{~kg} /\left(\mathrm{m}^{3} \cdot \mathrm{s}\right)$ \\
Consumption related to metabolism & $\gamma_{0}^{n l}$ & $7 \times 10^{-5}$ & $\mathrm{~kg} /\left(\mathrm{m}^{3} \cdot \mathrm{s}\right)$ \\
HCs-IF interfacial tension & $\sigma_{h l}$ & 72 & $\mathrm{mN} / \mathrm{m}$ \\
TCs-HCs interfacial tension & $\sigma_{t h}$ & 36 & $\mathrm{mN} / \mathrm{m}$ \\
TCs-IF interfacial tension & $\sigma_{t l}$ & 108 & $\mathrm{mN} / \mathrm{m}$ \\
Lysis parameter & $\lambda$ & 0.0 & - \\
\hline
\end{tabular}

Table 2. Parameters related to oxygen diffusion.

\begin{tabular}{cccc}
\hline Parameter & Symbol & Value & Unit \\
\hline Diffusion coefficient of oxygen in interstitial fluid & $D_{0}^{\overline{n l}}$ & $3.2 \times 10^{-9}$ & $\mathrm{~m}^{2} / \mathrm{s}$ \\
Coefficient $\delta$ & $\delta$ & 2 & - \\
Normal mass fraction of oxygen in tissue & $\omega_{\text {env }}^{\overline{n l}}$ & $4.2 \times 10^{-6}$ & - \\
\hline
\end{tabular}

Table 3. Parameters for ECM with porosity 0.5.

\begin{tabular}{cccc}
\hline Parameter & Symbol & Value & Unit \\
\hline Density of the solid phase & $\rho^{s}$ & $1 \times 10^{3}$ & $\mathrm{~kg} / \mathrm{m}^{3}$ \\
Poisson's ratio of the ECM & $v$ & 0.4 & - \\
Yield effective stress limit & $\mathrm{t}^{\mathrm{s}} \mathrm{eff}^{\prime} y$ & $5 \times 10^{1}$ & $\mathrm{~Pa}$ \\
Viscosity & $\eta$ & 5 & $\mathrm{~Pa} \cdot \mathrm{s}$ \\
Hardening modulus & $H$ & $1.0 \times 10^{2}$ & $\mathrm{~Pa}$ \\
\hline
\end{tabular}

As said above the ECM is treated as a deformable solid phase with a visco-elastic constitutive law. A different stiffness of the solid skeleton corresponds to a different volume fraction of the solid phase. In the cases with a nonuniform porosity, the following relations are assumed for the Young's modulus, the intrinsic permeability $k$ and the parameter $a$, which is a constant parameter related to ECM porosity, used in the saturation-capillary pressure relationship

$$
\begin{aligned}
E_{A} & =\frac{1-\varepsilon_{A}}{1-\varepsilon_{B}} E_{B} \\
k_{A} & =\left(\frac{\varepsilon_{A}}{\varepsilon_{B}}\right)^{3} k_{B} \\
a_{A} & =\left(\frac{\varepsilon_{A}}{\varepsilon_{B}}\right) a_{B}
\end{aligned}
$$

$A$ and $B$ refer to the cases with different porosity dealt with in Section 4.6. The Young's modulus $E=500 \mathrm{~Pa}$, the intrinsic permeability $k=1.8 \times 10^{-15} \mathrm{~m}^{2}$, and the coefficient $a=590 \mathrm{~Pa}$ of zone B, with porosity $\varepsilon=0.5$, are considered as the reference values.

Hence the Young's modulus for the zone where ECM porosity is $0.85,0.8,0.3$ is, respectively, $150 \mathrm{~Pa}, 200 \mathrm{~Pa}, 700 \mathrm{~Pa}$. 


\section{Results}

\subsection{Morphological and Mechanical Characterization of Hydrogels for the Inclusion of U87-MG Tumor Cells}

Collagen is the most ubiquitous protein in the ECM and forms random networks of fibers depending on the assembling conditions [38,39]. In the brain, collagen is mostly found along the vascular network together with other proteins, such as laminins and fibronectin, and provides mechanical support as well as important cues for tumor cell dynamics [3,14,17] Another major component of the brain ECM is hyaluronic acid. This is a naturally occurring glycosaminoglycan that has the capability to bind to cell specific surfaces receptors (CD44), which are necessary for cell anchorage, homeostasis, and proliferation [15,40-42]. For this, both COL and HA have been used in different forms to reproduce different portions of the brain tissue.

Cells of glioblastoma type IV transfected with green florescence protein-U87-MG GFP — were included into hydrogels of COL and HA either individually or as preformed spheroids (Figure 3).



Figure 3. Schematic representation of the experimental plan. Glioblastoma type IV cells transfected with green florescence protein (U87-MG GFP ${ }^{+}$) are encapsulated into 3D hydrogels, made out of collagen type I (COL) or hyaluronic acid (HA), either individually or as preformed spheroids. Representative images of the two different hydrogels and cell culturing conditions (suspension and spheroids, at day 4 of culture) are displayed on top and bellow, respectively. Some distinctive features of U87-MG spatial organization and morphology in the two different hydrogels are already visible in the four central insets: cell elongation and roundness; compact tumor core and infiltrating region.

For individual cells, these were first expanded on a dish, then resuspended and added to the natural polymeric hydrogel. For spheroids, cell clusters of about $200 \mu \mathrm{m}$ were preformed and eventually included in the polymeric hydrogel. Finally, the COL and HA mixtures were deposited at the bottom of polystyrene multiwell plates, precoated with agarose, polymerized, and left in cell culture media for up to 8 days.

Knowing that the microstructural and biomechanical features of the extracellular matrix may severely affect the growth and spreading of malignant tissues, the morphology and deformability of the hydrogel scaffolds were characterized. First, scanning electron microscopy (SEM) was used to assess the microstructure of COL and HA. Figure 4A and its lateral insets show the organization of collagen fibers within the hydrogel. COL fibers appear entangled, with a diameter of approximately 
$10 \mathrm{~nm}$, and randomly arranged to form a matrix with pores varying in size between about 20 and $70 \mathrm{~nm}$, as from SEM images. Differently, HA presents a more compact organization of thinner (about $5 \mathrm{~nm}$ ) and curly fibers (Figure 4B). Importantly, the magnified SEM image for HA does not reveal any obvious, regular porosity of the scaffold. This supports the notion by which HA hydrogels can be used for mimicking the soft and dense brain parenchyma, whereas fibers within COL tend to resemble morphological features of ordered structures in the brain [3]. Subsequently, the mechanical deformability of the hydrogels was assessed under wet conditions by standard compression testing. As expected, COL presented a higher Young's modulus $E$ as compared to HA. Specifically, as documented in the chart of Figure 4C, E was $5.16 \pm 1.40 \mathrm{kPa}$ for COL and $0.20 \pm 0.03 \mathrm{kPa}$ for HA, assuming a Poisson coefficient $v=0.45$. These values are in good agreement with previously reported characterizations for human brain tissue [43-45].

$$
\text { A }
$$


$\mathrm{B}$
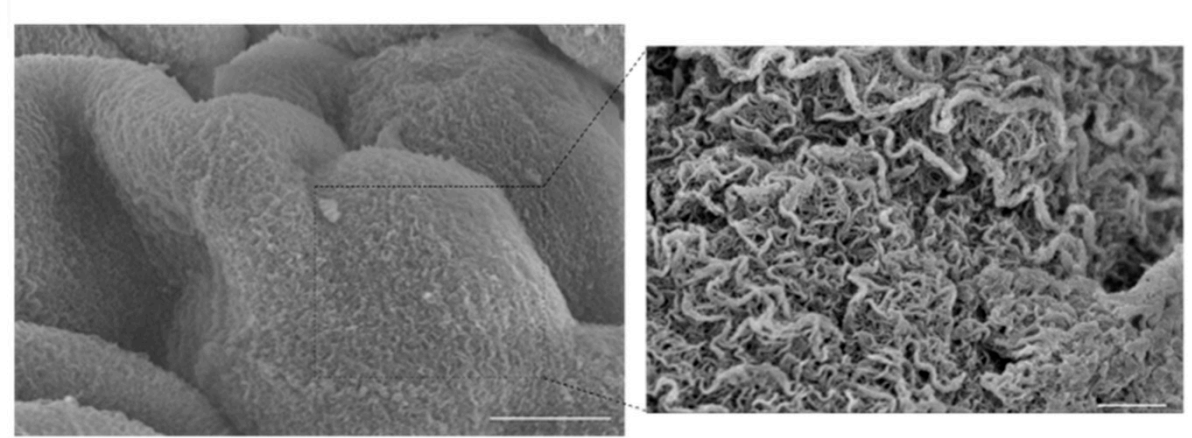

C

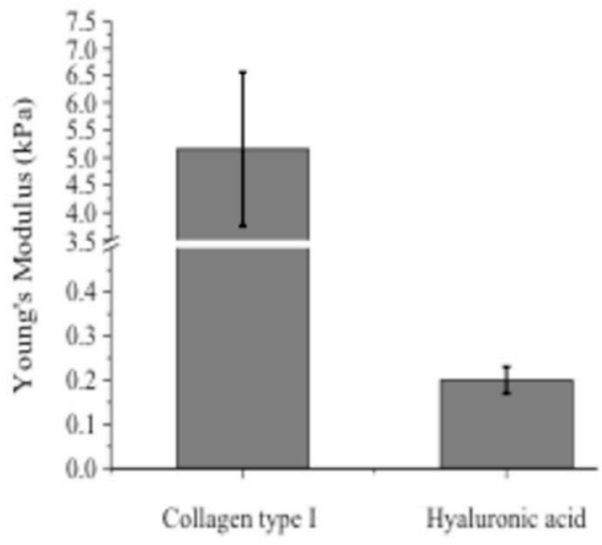

\begin{tabular}{c|c} 
Materials & $\begin{array}{c}\text { Young's Modulus } \\
(\mathrm{kPa})\end{array}$ \\
\hline $\begin{array}{c}\text { Collagen } \\
\text { type I }\end{array}$ & $5.16 \pm 1.04$ \\
\hline $\begin{array}{c}\text { Hyaluronic } \\
\text { acid }\end{array}$ & $0.20 \pm 0.030$ \\
\hline
\end{tabular}

Figure 4. Mechanical characterization of natural hydrogels. (A,B) SEM micrographs of COL and HA, respectively, showing very distinct morphologies. Magnified images are presented on the right. COL presents a fibrous structure with a variable porosity. HA presents a complex dense structure. (C) Young's modulus of COL and HA hydrogels. Scale bar $=5 \mu \mathrm{m}$ and $500 \mathrm{~nm}$ for the low and high magnification images, respectively. Statistical analysis was determined using $t$-test multiple comparison with a confidence level of $95 \%(p<0.05)$. GraphPad was used. 


\subsection{Growth of Individual U87-MG Cells in Different Hydrogels}

In studying the proliferation and migration ability of cells within man-made scaffolds, it is fundamental to confirm cell growth over time. Fluorescent and electron scanning microscopy together with MTT assays were performed to document GBM cell proliferation and morphology within COL and HA.

Fluorescent images of U87-MG GFP ${ }^{+}$cell dispersions in COL (Figure 5A) and in HA (Figure 5B) were acquired from day 1 to day 7 , post seeding. In both cases, the fluorescent signal significantly increased over time and spreads quite uniformly over the entire hydrogel, thus documenting cell proliferation. Fluorescent images for all time points, namely days 1,3,5, and 7, are shown in Figure 6 . Cell proliferation via MTT analysis is shown in Figure 7.
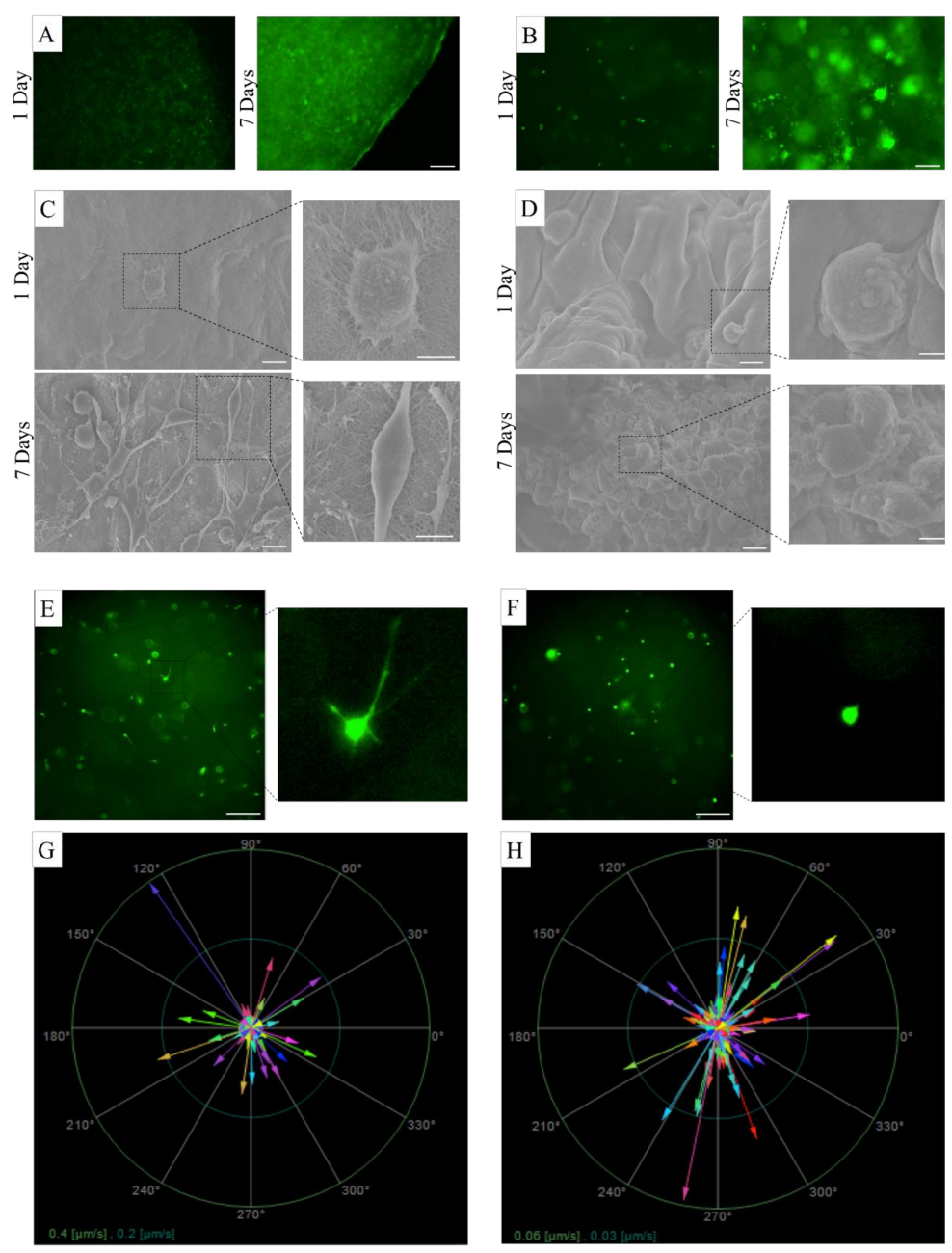

Figure 5. Behavior of individual glioblastoma type IV cells in hydrogels. (A,B) Fluorescent microscopy images of U87-MG GFP ${ }^{+}$cells at days 1 and 7 post inclusion in COL and HA, respectively. Increase in fluorescent intensity indicates cell proliferation (scale bar $=100 \mu \mathrm{m}) .($ C,D) SEM images of U87-MG cells at days 1 and 7 post inclusion in COL and HA, respectively. High magnification micrographs, on the right, document the different cell morphologies: elongated and separated in COL; rounded and clustered in HA (scale bar $=10 \mu \mathrm{m}$ ). (E,F) Fluorescent microscopy images of U87-MG cells included in $\mathrm{COL}$ and $\mathrm{HA}$, respectively. High magnification images, on the right, confirm elongated morphologies for COL and rounded shapes for HA (scale bar $=100 \mu \mathrm{m}) .(\mathbf{G}, \mathbf{H})$ Polar diagrams representing cell mobility behavior of U87-MG cells included in COL and HA, respectively. 


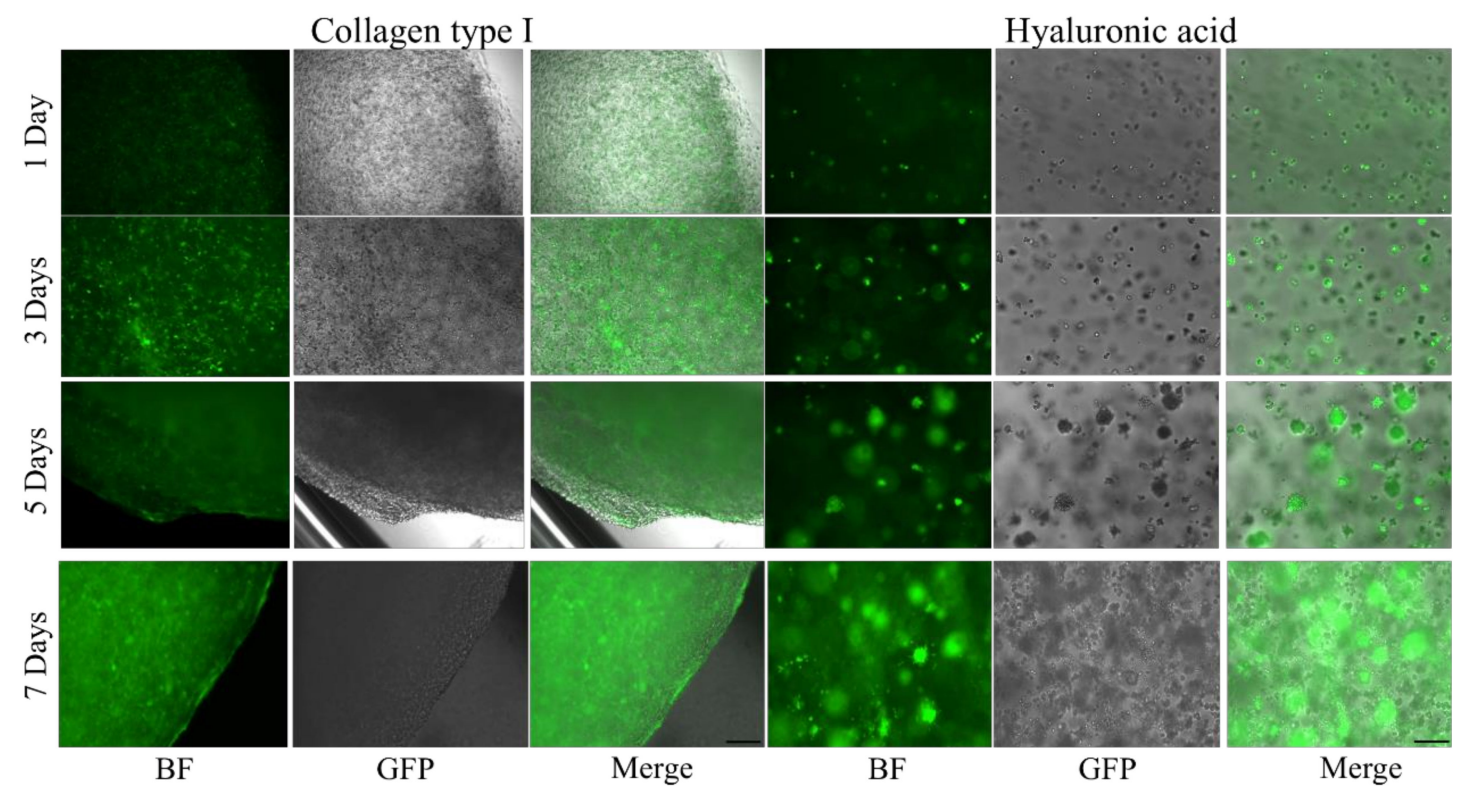

Figure 6. Cell morphology and spatial distribution. Morphology and spatial distribution of U87-MG cells, within the two different scaffolds, were examined via fluorescent microscopy at several time points, namely at 1, 3, 5, and 7 days post inclusion. Scale bar $=100 \mu \mathrm{m}$.
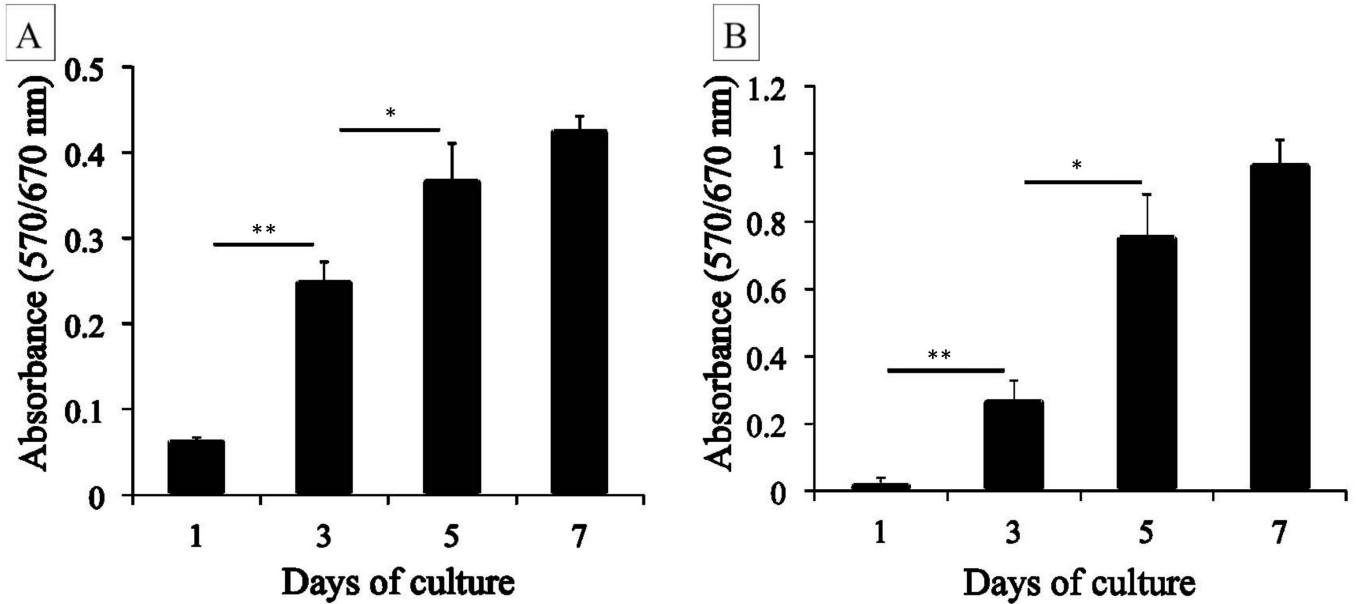

Figure 7. Cell proliferation. Continuous growth of U87-MG cells was reported throughout the culture period, within both scaffolds: (A) Collagen and (B) Hyaluronic acid. In particular, proliferation was statistically significant for the first three time points, namely from day 1 to $3^{* *}(p=0.0001$ for COL; $p=0.002$ for HA); from day 3 to $5 *$ ( $p=0.0126$ for COL; $p=0.035$ for HA), whereas no significant difference was observed between the last two time points, corresponding to days 5 and 7 . Statistical analysis was performed using $t$-test multiple comparison with a confidence level of $95 \%$ $(p<0.05)$. GraphPad was used.

\subsection{Cell Imaging}

Over the course of the culture, spatial distribution and morphology of encapsulated U87-MG GFP ${ }^{+}$ cells within collagen type I and hyaluronic acid hydrogels was monitored using a fluorescent inverted microscope (Leica DMI6000 B) equipped with a high-speed camera (Leica DFC360 FX). Images were acquired by the use of bright field and $488 \mathrm{~nm}$ filters. Images were obtained at $10 \times$ magnification. 


\subsection{MTT Assay}

The proliferation of encapsulated U87-MG cells within both gels was evaluated at different time points, using thiazolyl blue staining (MTT, Sigma-Aldrich), in which absorbance data correlate to cell metabolic activity. In brief, at each time point, the medium was removed and replaced with $1 \mathrm{~mL}$ fresh medium supplemented with MTT stock solution at a final concentration of $5 \mathrm{mg} / \mathrm{mL}$. After $3 \mathrm{~h}$ incubation, medium was removed and the converted dye was solubilized by the addition of $1 \mathrm{~mL}$ ethanol absolute. To obtain a homogenous solution, hydrogels were disrupted mechanically via a potter [29]. Absorbance was measured at $570 \mathrm{~nm}$ with background subtraction at $670 \mathrm{~nm}$.

With time, cells were also observed to change their morphology as they were proliferating and moving about the hydrogels. This is clearly documented by SEM images in Figure 5C,D, respectively, for COL and HA. Initially, U87-MG cells presented a rounded shape on both scaffolds. However, at later time points, cells cultured in COL were mostly elongated presenting a bipolar, spindle-shaped morphology, whereas cells cultured in HA mostly preserved the original rounded shape. Figure 8 shows similar morphologies for U87-MG cells in COL already at day 3. It should also be noted that U87-MG cells in HA have been observed to cluster together forming larger aggregates, differently from cells in COL which are rather isolated. This is already evident at day 3 as evidenced by the right column images representative of higher magnifications of those on the left side (Figure 8). This behavior is also confirmed by the fluorescent images presented in Figure 5E,F. Again, U87-MG cells cultured in COL tend to assume an elongated morphology, which preludes to higher motility and migration potential. Differently, the same cells in HA hydrogels assume a rounded shape and are more prone to clustering as shown in the high magnification images (right column) of Figure 8.

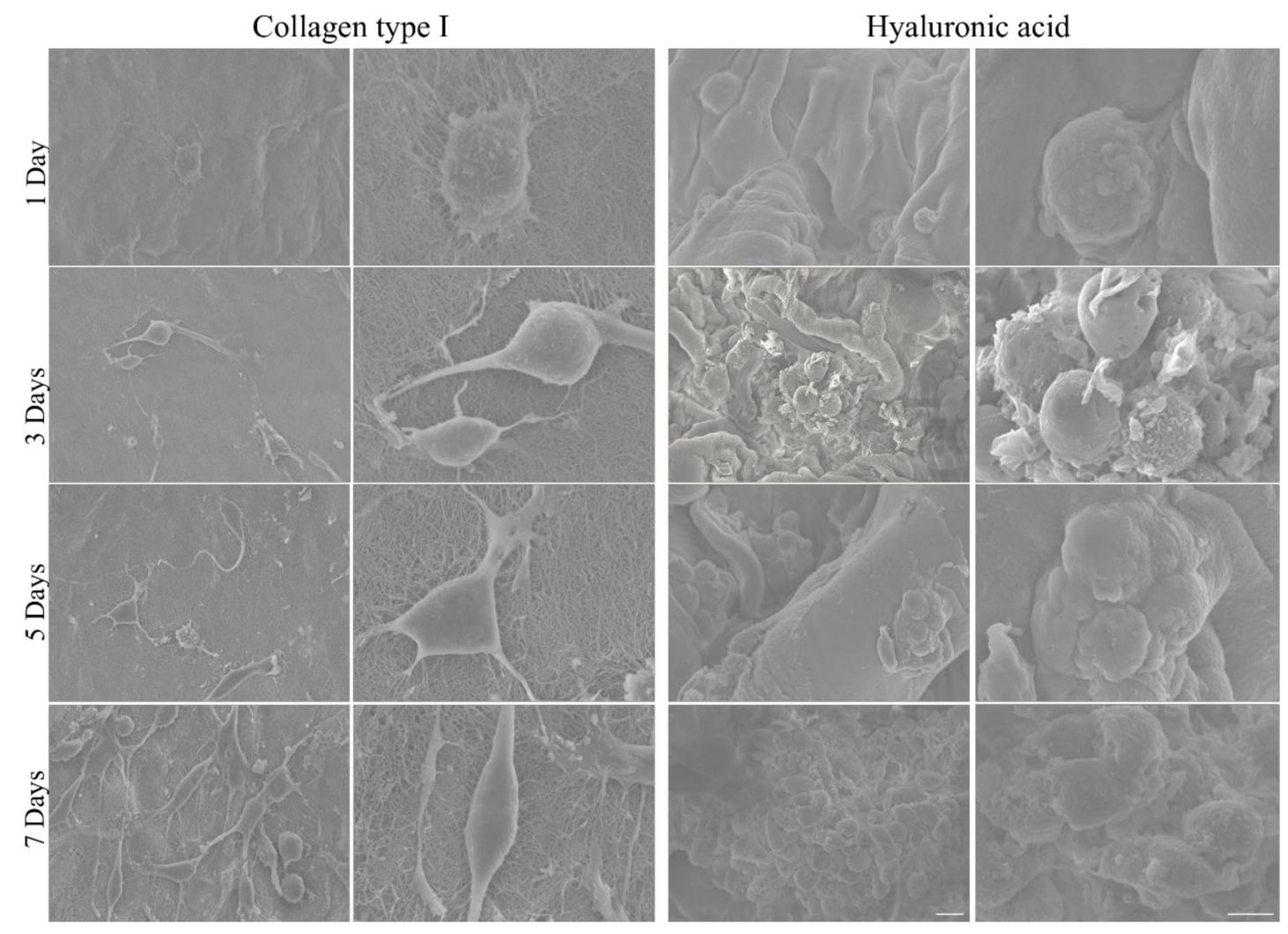

Figure 8. Cell morphology within the scaffolds. Morphology of U87-MG cells was monitored over time, from day 1 to 7 , via scanning electron microscopy. Cells included in COL gels were observed to alter their initial rounded shape into a more spindle-like, elongated morphology. This behavior is already observed at earlier time points. Cells included in HA gels grew into small clusters preserving their original rounded shape. Images on the right column panel of each gel (Collagen type I and Hyaluronic acid) represent higher magnifications of the related left ones. Scale bar $=50 \mu \mathrm{m}$. 
To further characterize the hydrogel-dependent cell migration behaviors, individual U87-MG $\mathrm{GFP}^{+}$cells were monitored by registering their position and measuring the length of the path traced by cell nuclei over time. Tumor cells in COL hydrogels migrated over longer distances as compared to cells in HA, within the same time period. This is reflected by the polar diagrams of Figure 5G,H and in the Supporting Movies S1 and S2. These different cell behaviors are in agreement with previous reports showing that HA favors the formation of cell clusters [14], whereas COL supports more cell spreading [46].

\subsection{Growth of U87-MG Spheroids in Different Hydrogels}

Cellular spheroids of glioblastoma type IV were preformed into GravityTRAP ${ }^{\mathrm{TM}}$ ULA plates, via the progressive assembling of individual U87-MG cells. After 1 day, these preformed spheroids, presenting a radius of about $200 \mu \mathrm{m}$, were included in the natural polymeric hydrogels and monitored over time for their growth and spatial organization, from $18 \mathrm{~h}$ up to 8 days post inclusion.

Figure 9A,B shows bright field and fluorescent images of tumor spheroids at different time points for COL and HA, respectively. Five spheroids were used under each condition. Interestingly, these figures show that spheroids included into COL rapidly tend to spread with individual cells rapidly moving about the scaffold already at day 1 . This can be readily observed in the bright field and fluorescent images were the high-cell density circle (dark area), which corresponds to the Tumor Core region, is surrounded by a low-cell density corona (bright area), which corresponds to the Tumor Infiltrating region. A similar trend is also observed for the HA case, but it can only be appreciated at later time points, after day 6 . The overall radius of the tumor spheroids, including the radius of the core and the thickness of the surrounding infiltrating region, and the radius of the tumor core are charted in Figure 9C,D for the different times points, as measured via fluorescent microscopy, respectively, for COL and HA. For spheroids growing in COL (Figure 9C), the overall radius varies from about $200 \mu \mathrm{m}$ at time of encapsulation to $1221.88 \pm 22.93 \mu \mathrm{m}$ at day 8 , documenting a 6 -fold increase. The high-cell density tumor core radius reaches $551.22 \pm 52.56 \mu \mathrm{m}$ after 8 days of culturing, with a 2.5-fold increase. This data also shows how significant the spreading of U87-MG cells within COL is. As reported in Figure $9 \mathrm{C}$, the overall tumor radius, comprising the surrounding infiltrating region, is roughly two times that of the tumor core for the whole observation period, starting already at $18 \mathrm{~h}$. On the other hand, spheroids growing inside HA were observed to grow from about $200 \mu \mathrm{m}$ at time of encapsulation to $783.01 \pm 145 \mu \mathrm{m}$ at day 8 , documenting only a 3.5-fold increase (Figure 9D). Additionally, the high-cell density tumor core radius reaches $448.27 \pm 69.53 \mu \mathrm{m}$ after 8 days of culturing, with a 2.5-fold increase. Only between day 3 and day 4, an infiltrating region was observed. This data mostly indicates a delayed and overall lower growth of the infiltrating region for the spheroid cultured in HA as compared to COL. Overall this different behavior in tumor spheroid growth and infiltration appears to match with the different behavior registered for the individual tumor cells (Figure 5), whereby U87-MG in COL mostly take, already after a few hours of culturing, an elongated shape priming towards migration whereas the same cells in HA acquire a rounded shape prone to clustering.

\subsection{Predicting the Growth of U87-MG Spheroids in Different Scaffolds}

The multiphysics computational model described in Section 3, is used to predict the temporal development of GBM spheroids within different microenvironmental conditions.

The role of two major mechanical parameters of the hydrogels was systematically assessed, namely the Young's modulus $E$ and the porosity $\varepsilon$, defined as $1-V_{s} / V_{\text {tot }}$ where $V_{s}$ is the volume occupied by the solid porous ECM within the whole domain $V_{\text {tot }}$. Therefore, the larger $\varepsilon$ is and the smaller the volume fraction occupied by the solid ECM is, the higher is the porosity of the ECM. For HA, a Young's modulus of $200 \mathrm{~Pa}$ was considered (see Figure 2) while the matrix porosity $\varepsilon$ was varied between 0.8 and 0.3 , and kept homogeneous across the whole domain surrounding the initial spheroid. 
A

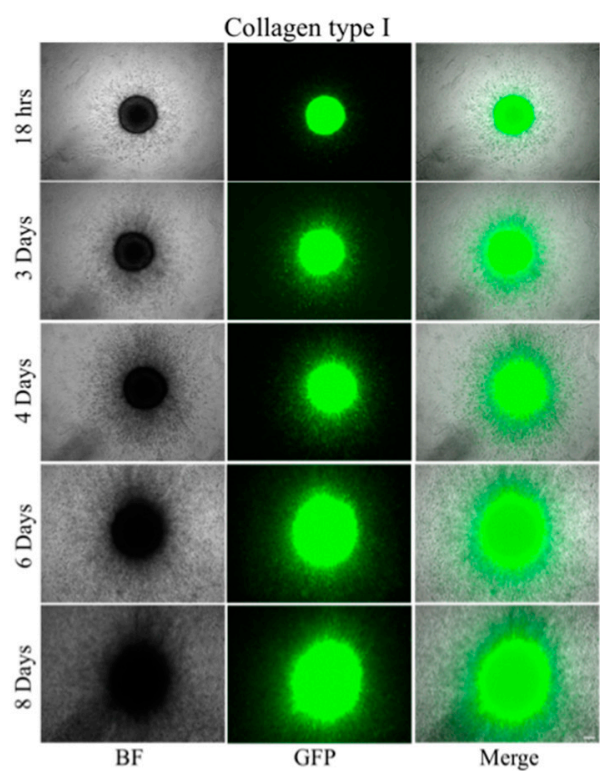

$\mathrm{C}$



B



$\mathrm{D}$

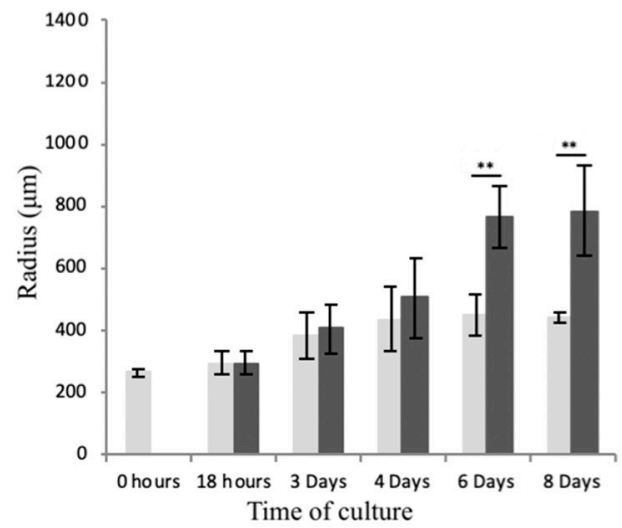

Figure 9. Behavior of glioblastoma type IV spheroids in hydrogels. (A,B) Microscopy images-bright field (BF), fluorescent (GFP), and merged - of U87-MG GFP ${ }^{+}$spheroids documenting tumor growth over time $\mathrm{COL}$ and $\mathrm{HA}$, respectively. The tumor mass appears as a dense (high fluorescence intensity) inner portion—core-surrounded by a lighter (low fluorescence intensity) outer portion—infiltrating region (scale bar $=50 \mu \mathrm{m})$. $(\mathbf{C}, \mathbf{D})$ Tumor spheroid progression over time, expressed in terms of overall radius of the tumor mass (grey bars) and radial dimension of the infiltrating region (dark bars) for COL and HA, respectively. Statistical analysis was done using $t$-test multiple comparison with a confidence level of $95 \%(p<0.05)$. GraphPad was used.

Figure 10A shows the variation of the predicted tumor core size as a function of time for different values of $\varepsilon$. For $\varepsilon=0.8$, a good agreement $\left(R^{2}=0.78\right)$ was found between the computational (solid line) and the experimental data for HA (dots). On the other hand, as the porosity $\varepsilon$ reduces, a significant decrease in tumor growth rate was observed. At 8 days, the tumor size for $\varepsilon=0.5$ and 0.3 was, respectively, $18.52 \%$ and $39.48 \%$ smaller as compared to the $\varepsilon=0.8$ case ( 389.62 and $289.35 \mu \mathrm{m}$ as compared to $478.18 \mu \mathrm{m}$, respectively). Indeed, dense matrices (low $\varepsilon$ ) favor pressure build-up within the porous solid which eventually limits cell proliferation and induces cell death. 
A



B



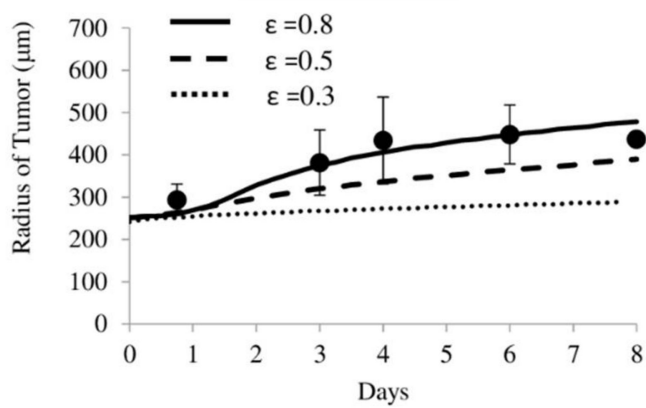

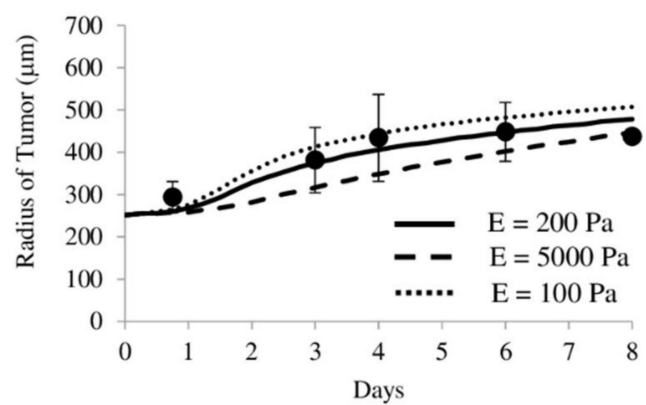

$\mathrm{C}$

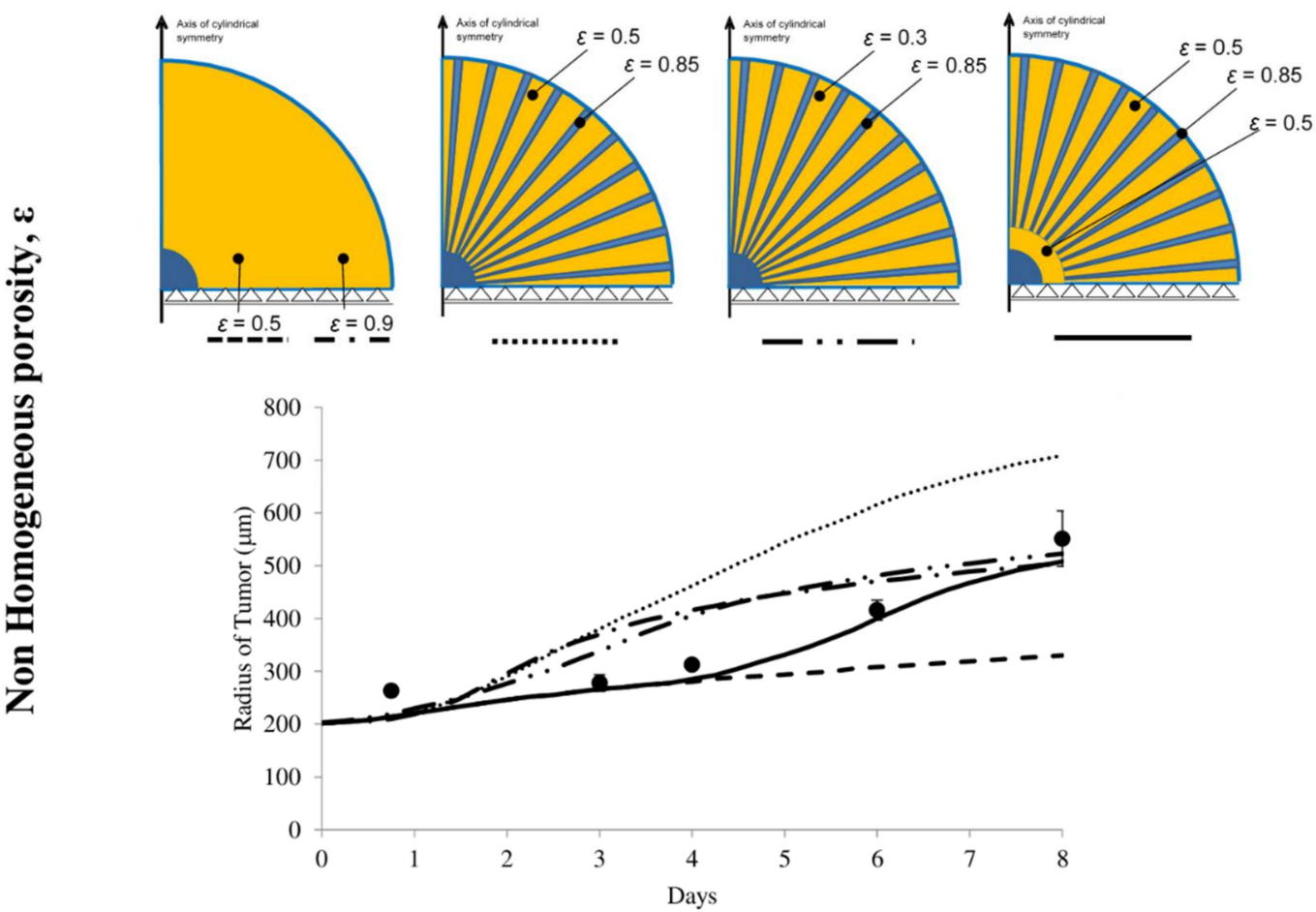

Figure 10. Computational model predictions for tumor spheroid growth. (A) Tumor core radius variation over time for different ECM porosity conditions, ranging from high porosity $(\varepsilon=0.8)$ to low porosity $(\varepsilon=0.3)$. ECM properties are homogenous across the domain, externally to the central blue core. (B) Tumor core radius variation over time for different ECM Young's moduli $E$, ranging from a soft $(E=100 \mathrm{~Pa})$ to a rigid $(E=5000 \mathrm{~Pa})$ situation, for a constant porosity $\varepsilon=0.8$. ECM properties are homogenous across the domain, externally to the central blue core. (C) Tumor core radius variation over time for different spatial organizations of the ECM porosity, namely uniform (left schematic), nonuniform with alternating radial sectors of different porosities (two middle schematics), and a combination of the previous two configurations (right schematic). The legend for the graph lines is given under the corresponding images. In the graph, continuous lines show numerical results, dots report the average values of the experimental tests (the error bars show the standard deviation from the average values). 
Then, starting from the conditions resembling the growth of the tumor core in HA, the effect of the Young's modulus $E$ was investigated. Figure 10B reports on the tumor growth over time for $E$ ranging between 100 and $5000 \mathrm{~Pa}$, the latter being the Young's modulus measured for COL (see Figure 4). As expected, an increase in matrix rigidity leads to a decrease in tumor growth. Indeed, following the same argument presented above for $\varepsilon$, a higher mechanical constriction results in lower cell proliferation. Notably, despite the huge variation in Young's modulus, from 100 to $5000 \mathrm{~Pa}$, the corresponding change in tumor size is quite moderate. At 8 days, the tumor size for $E=100$ and $5000 \mathrm{~Pa}$ was, respectively, $6.07 \%$ higher and $6.74 \%$ lower as compared to the $E=200$ Pa case (507.25 and $445.95 \mu \mathrm{m}$ as compared to $478.18 \mu \mathrm{m}$, respectively). This evidently implies that tumor growth is more sensitive to variations in matrix porosity rather than rigidity. For the $E=200 \mathrm{~Pa}$ case there is a good agreement between computational results (solid line) and the experimental data (dots).

As shown in Figure 9, tumors in COL tend to grow faster than in HA. Therefore, in the numerical simulations, matrix porosity was changed to reproduce this behavior. In Figure 10C, the dashed and dash-dot lines reproduce the cases for $\varepsilon=0.5$ (low porosity) and $\varepsilon=0.9$ (high porosity). As expected, lower porosities were associated with smaller tumor sizes over time. However, in both cases, the computational predictions were far from the experimental data. Thus, since COL are characterized by fibers along which tumor cells can more easily migrate, it was assumed that the matrix surrounding the initial spheroid could be characterized by alternating radial sectors of low $(\varepsilon=0.5$ and 0.3$)$ and high $(\varepsilon=0.85)$ porosities. This configuration should favor tumor growth along the low porosity sectors as compared to the denser matrix regions. The dotted and dash-two dots lines in Figure 10C present data for these nonuniform porosity configurations. Within the first 3 days, both cases presented a similar behavior, with almost overlapping tumor growth curves. However, at longer time points, both cases predicted a faster growth as compared to the experimental data. Interestingly, at longer time points, the $\varepsilon=0.5 / 0.85$ case showed a trend similar to the experimental data but shifted towards larger sizes. As detailed in Figure 11, the dual porosity configurations $(\varepsilon=0.3 / 0.85$ and $\varepsilon=0.3 / 0.5)$ predicted quite similar tumor growth behaviors, with the latter giving overall smaller tumor volumes because of the overall lower porosity of the matrix. Both simulations with dual configuration of porosity are not able to reproduce the experimental data (dots). More interestingly, the $\varepsilon=0.3 / 0.85$ case resulted in tumor core volumes very similar to those estimated in the case of a uniformly porous matrix with $\varepsilon=0.9$ (high porosity) (Figure 10C). These numerical results would imply that matrices with nonuniform porosities experience a more rapid tumor growth as compared to uniform and higher porosity matrices. However, none of the two general porosity configurations so far considered (uniform and nonuniform with radial sectors) accurately predict the tumor growth in COL. This is mostly because of the relatively low growth rate observed within the first 4 days of culturing. Therefore, the more realistic configuration for tumors growing in COL resulted in a combination of the above cited two cases: a rim with uniform porosity surrounding the initial tumor spheroid with $\varepsilon=0.5$, which serves to modulate the initial growth rate; and alternating radial sectors of low $(\varepsilon=0.5)$ and high $(\varepsilon=0.85)$ porosities, characterizing the most exterior portion of the matrix. This case is identified as $\varepsilon=0.5+0.5 / 0.85$ in the following. For this configuration, a good agreement $\left(R^{2}=0.91\right)$ was achieved between the computational (solid line) and the experimental data (dots) for COL, as documented in Figure 10C. Interesting is comparison of the resulting distribution of the tumor volume fractions for the cases $\varepsilon=0.3 / 0.85$ and $\varepsilon=0.5+0.5 / 0.85$ after 8 days of simulated growth, drawn in Figure 12. Since tumor growth influences the porosity, the porosity distribution after 8 days is shown as the second figure for each case. The initial porosity distribution can be seen in Figure 10C. A more developed heterogeneity of the tumor cells distribution appears clearly in Figure 12B in line with what was expected from the porosity distribution of COL shown in Figure 4A. The blue part is the remaining region of the simulated domain not yet occupied by the core. It contains however the corona. 

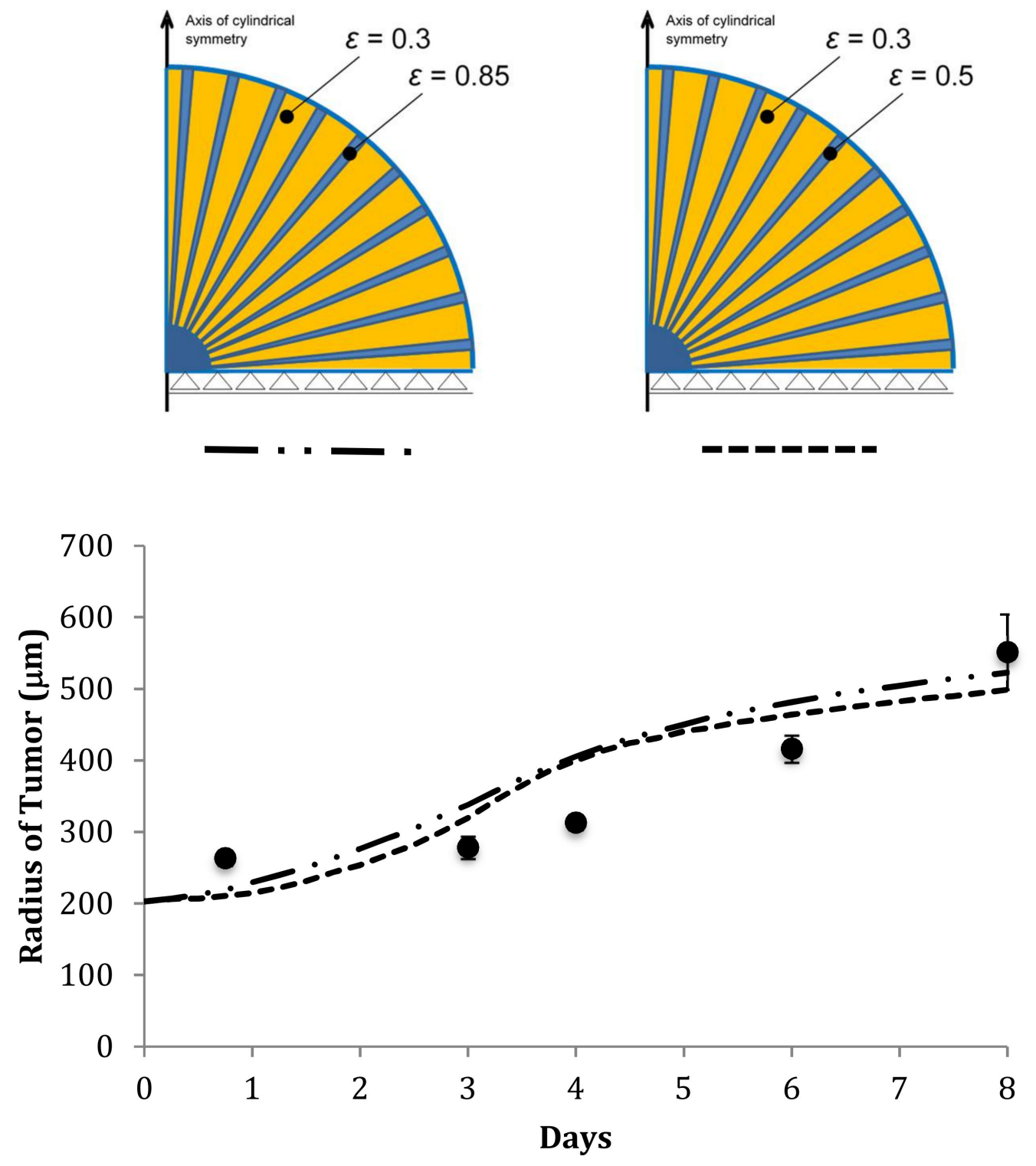

Figure 11. Radius of the tumor against time for different ECM porosity distributions. Initially, the tumor spheroid is located within the central blue area, with $\varepsilon=0.85$ (low ECM content). Externally at this central area, ECM has a nonhomogeneous porosity characterized by alternating radial sectors with $\varepsilon=0.3$ and $\varepsilon=0.85$ (dashed-two dots line) and $\varepsilon=0.3$ and $\varepsilon=0.5$ (dashed line). The two configurations provide similar results in terms of tumor growth. However, the configuration with the overall lowest porosity $(\varepsilon=0.5 / 0.3)$ predicts a slightly lower tumor growth. Dots represent the same average values of the experimental data and related error bars of Figure 10C. 

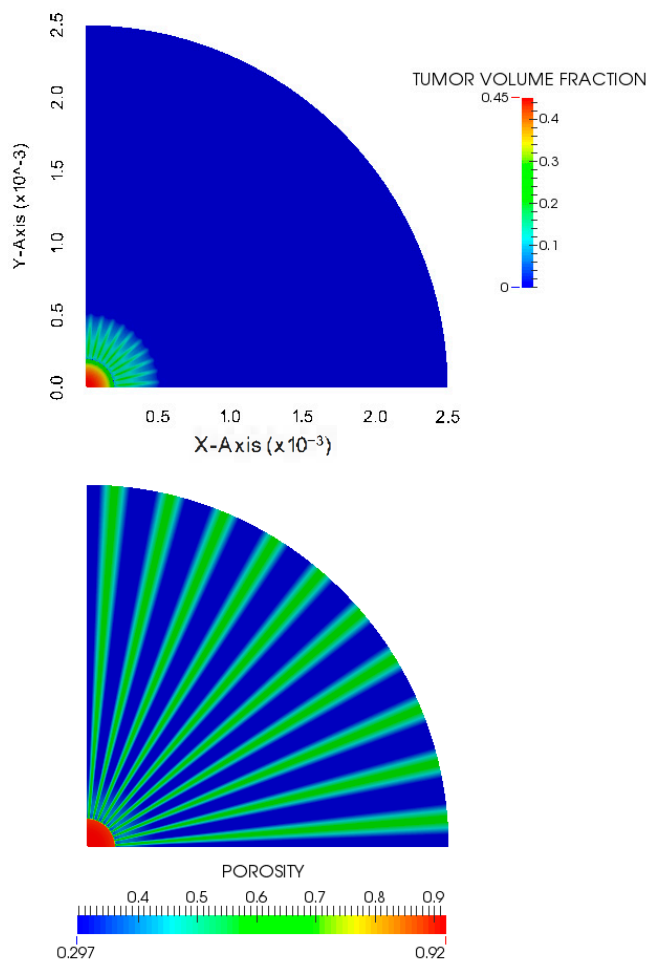

(A)
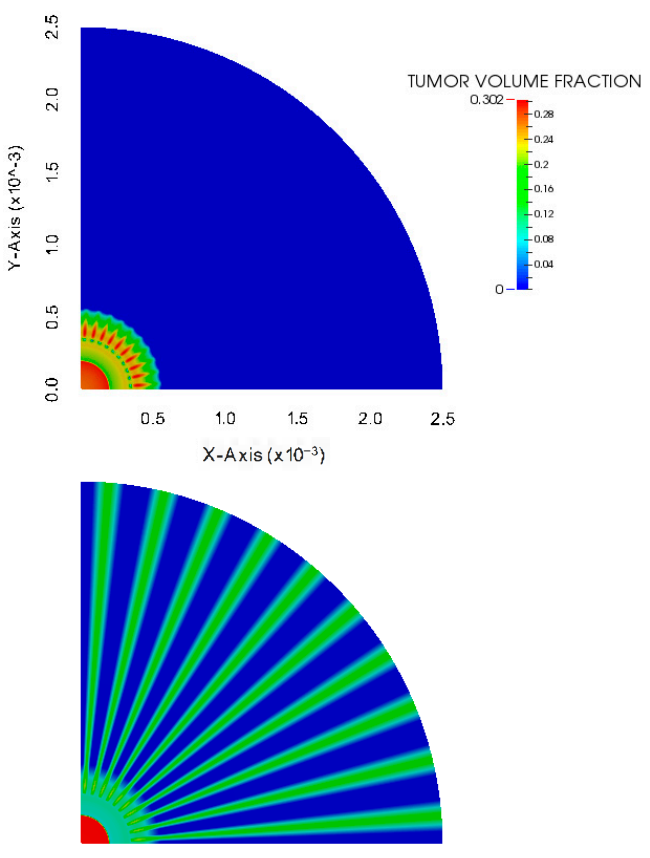

POROSITY



(B)

Figure 12. Tumor core after 8 days of growth for different ECM porosity distributions. (A) (top) Spatial distribution of tumor cells volume fraction in the tumor core for the porosity configuration $\varepsilon=0.3 / 0.85$ after 8 days; (below) porosity distribution at the same time instant. (B) (top) Spatial distribution of tumor cells volume fraction in the tumor core for the porosity configuration $\varepsilon=0.5+0.3 / 0.85$ after 8 days; (below) porosity distribution at the same time instant. A more developed heterogeneity can be seen in (B), in line with what was expected from Figure 4A. The outer part is occupied by the corona which has a much lower density and does not appear in the image. 


\section{Discussion}

In this work, the proliferation and migration propensity of human glioblastoma type IV cells (U87-MG) was studied within different tridimensional microenvironments: hyaluronic acid hydrogels (HA), whose density and rigidity resembled the gel-like properties of the brain parenchyma; collagen hydrogels (COL), whose spatial organization with fibers and pores resembled the ordered structures in the brain, such as the perivascular space and axonal fibers. Although none of these two simple hydrogels can precisely mimic the tridimensional complexity of a human brain tissue, relevant cell features, such as morphology, migration, clustering, and proliferation, were found to closely match in-vivo observations. In particular, glioma cells tend to spread more rapidly in matrices with organized fibers, such as COL, whereas cell clustering is typical of dense and poorly organized matrices, such as HA. This was documented in Figure 5. It was noticed that individual U87-MG cells rapidly assume an elongated shape, with cell membrane ruffling and long filopodial protrusions, on COL. This spindle shaped cell morphology is a clear sign of migratory phenotype and was observed as early as a few hours post-inclusion. Very differently, individual tumor cells on HA preserve their original rounded shape, which is more prone to form large and dense cell clusters. The different cell morphology could indeed be associated with ECM rigidity, as previously documented by Kumar and collaborators [47], which might induce a cell epithelial to mesenchymal transition and facilitate migration [48]. Furthermore, by tracking over time the motion of individual cell nuclei, U87-MG cells were confirmed to have higher motility and travel longer distances on COL as compared to HA. Overall this behavior agrees with in-vivo observations and the notion that glioma cells would more efficiently migrate along organized brain structures (blood vessel walls and axonal fibers) rather than across the dense extracellular space [3,49-51].

This individual cell behavior is then reflected in the overall development of neoplastic masses, as documented in Figure 9. Tumor spheroids included into COL gels grow at faster rates and, more importantly, infiltrate more deeply the surrounding matrix, as compared to HA gels. At 8 days post spheroid inclusion, neoplastic masses in COL have a more developed infiltrating front as compared to HA. On the other hand, tumors in HA present a more dense and smaller infiltrating front again suggesting that cell clustering is preferred to individual cell migration within such matrices. This can be readily appreciated by comparing the individual pictures in Figure 9A,B: the infiltrating front is more diffuse and spread over the entire region of interest in the case of COL (Figure 9A), whereas it appears more compact and well defined in the case of HA (Figure 9B). At 8 days, the radius of the HA infiltrating front is still about two times smaller than for COL: the characteristic sizes are $345.55 \pm 139.09 \mu \mathrm{m}$ for HA and $661.65 \pm 39.68 \mu \mathrm{m}$ for COL. Supporting Movies S3 and S4 readily depict all these differences between COL and HA.

It is worth noting the different evolution in time of the tumor core growing in collagen type 1 and hyaluronic acid: in the first case there appears an accelerated trend while in the second case some tendency to a stable state result. In the medium the growth pattern appears linear. This is more clearly shown in Figure 13, where a direct comparison with spheroids growing in cell culture media is presented too. It is evident that spheroids in suspensions do not develop as rapidly and aggressively as spheroids embedded into more realistic environments: at 8 days, tumor spheroids developing in media have roughly half the size of tumors growing in gels $(551.22 \pm 52.56 \mu \mathrm{m}$ in COL, $448.27 \pm 69.53 \mu \mathrm{m}$ in HA, and $278.63 \pm 17.08 \mu \mathrm{m}$ in medium). Obviously, spheroids in suspension cannot develop a truly infiltrating front. This data continues to confirm that cell adhesion and interaction with proper extracellular matrices favor cell duplication and spreading. 
A

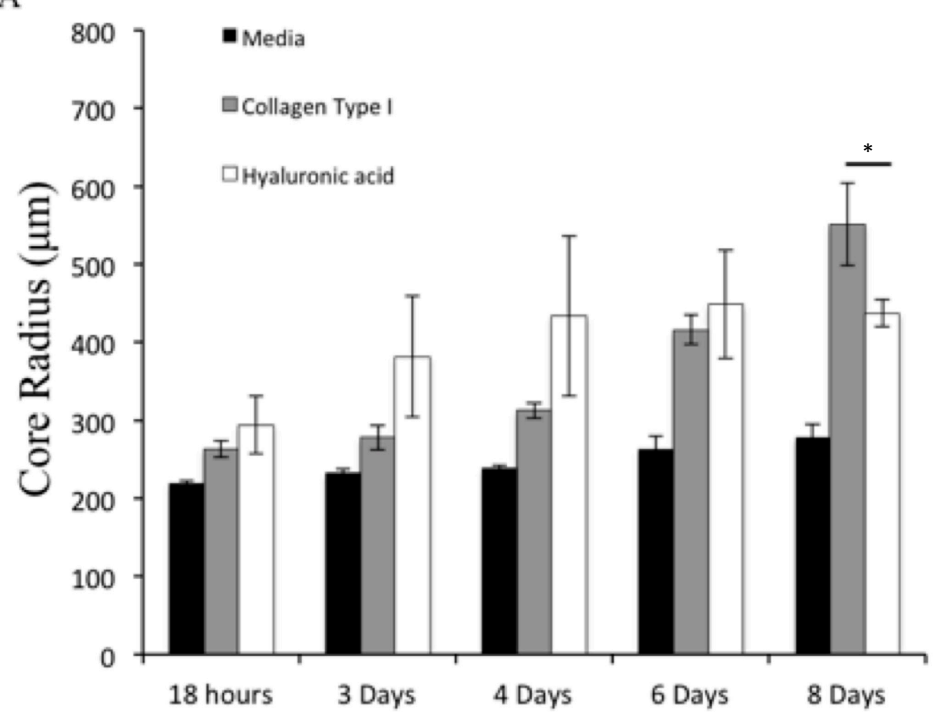

B

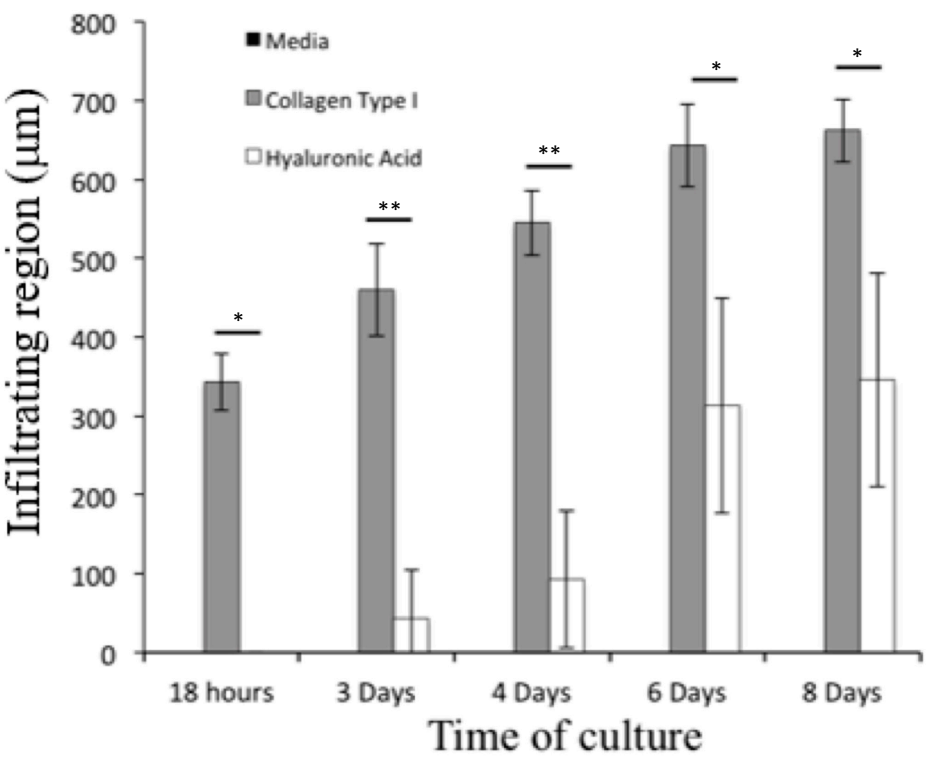

Figure 13. Tumor spheroid growth over time under different conditions. Variation of the tumor core radius (A) and infiltrating region size (B) over time for spheroids suspended in free medium (black bars), in COL hydrogels (gray bars) and in HA hydrogels (white bar). Note that no infiltrating region can be detected in the case of spheroids growing suspended in free medium. Statistical analysis was undertaken using $t$-test multiple comparison with a confidence level of $95 \%(p<0.05)$. GraphPad was used.

Finally, a multiphysics computational model was demonstrated to accurately predict the temporal growth of the tumor masses for both hydrogel configurations. A soft matrix with uniform porosity showed to give good results for spheroids grown in HA, whereas a nonuniform porosity distribution was required in order to match more closely the experimental data for spheroids cultivated in COL gels. Interestingly, simulations showed that nonhomogeneous porous matrices, modeled by alternating radial sectors with different values of porosity, could more efficiently support the spreading and growth of tumor masses as compared to uniform porosity matrices. This numerical evidence shows that the computational model is able to capture the higher migratory attitude of glioma cells in matrices with a random network of fibers as compared to matrices with a uniform dense structure. Further, the numerical simulations enable one to evaluate the influence of tumor growth on porosity, as 
illustrated in Figure 12 at day 8 as an exemplifying case. It is here important to note that the parameters for cell proliferation, adhesion, and ECM remodeling were kept identical for both hydrogel conditions. The in silico tests were performed by varying only the elastic modulus $\mathrm{E}$, the value of porosity $\varepsilon$, and its spatial organization. This confirms that major differences in tumor growth between the two gels have to be ascribed to mechanical cues, such as porosity and rigidity.

\section{Conclusions}

One of the key issues in glioblastoma progression is the highly infiltrating nature of the tumor mass, which is pathway dependent and marked by the ability of glioma cells to adopt different migratory strategies. In this work, for the first time, a set of quantitative studies was carried out, by means of in vitro and in silico tests, to elucidate the role of matrix porosity, rigidity, and morphology in the development of glioblastoma malignancy. Proliferation and motility of glioblastoma type IV cells were assessed in different extracellular matrices, namely made out of collagen type 1 and hyaluronic acid. Collagen matrices presented organized fibrous structures with a pore size ranging between 20 and $70 \mathrm{~nm}$ and a Young's modulus of $5000 \mathrm{~Pa}$. Differently, hyaluronic acid matrices appeared as a compact and disorganized cluster of short fibers with no obvious porosity and a Young's modulus of $200 \mathrm{~Pa}$. Glioblastoma type IV cells were observed to readily proliferate in both matrices, under similar culturing conditions. However, individual cells in collagen matrices exhibited an elongated morphology, similar to fibroblasts, documenting a propensity to migration whereas cells in hyaluronic acid matrices appeared globular. Culturing of tumor spheroids into the two different hydrogel matrices recapitulated the behavior manifested by individual cells. Glioblastoma type IV spheroids were documented to grow more rapidly in collagen matrices forming already after a few hours cell protrusions, possibly aligned with the collagen fibers. On the other hand, the same spheroids mostly developed as compact masses when cultured in hyaluronic acid matrices. Although infiltrating regions around the tumor core were observed for both matrices, this developed earlier and faster for spheroids included in collagen as compared to hyaluronic acid. A tumor growth multiphysics model, accurately predicting the different experimental behaviors in locally homogeneous environments, was able to match also the evolution in time of the tumor spheroids growing in the two different scaffolds by introducing some matrix heterogeneity: the plateau following the initial growth in hyaluronic acid and the faster trend in collagen. The computational results matching the realistic cases as well as those with different porosity morphology performed for investigative aim, confirmed that matrices with nonuniform porosities accelerate the development and tissue infiltration of neoplastic masses. All together, these data demonstrate that mechanical features of extracellular matrices, such as porosity and its spatial organization, are more relevant than matrix rigidity in supporting rapid tumor growth and infiltration. This experimental-computational framework can be used to quantitatively study the influence of porosity in tumor growth and to clarify the role of porosity morphology in the evolution of malignant masses. Furthermore, in silico tests allow to investigate many combinations of parameters and configurations, even in cases which would be impossible to reproduce in the laboratory. By exploiting the synergy of the tests performed in vitro and in silico, it is possible to predict the behavior of the neoplastic masses and their evolution over time, as well as to elucidate which are the most relevant biomechanical cues for combined therapies aiming simultaneously at killing cancer cells and modulating the tumor microenvironment. Finally, these first results about the importance of heterogeneity point to the need of investigating the impact of the stochastic heterogeneity of physical parameters on tumor growth because this heterogeneity is frequently attributed only to genetics $[52,53]$. This paper has shown that this is not necessarily the case and paves the way to further investigation on this aspect.

Supplementary Materials: The following are available online at http://www.mdpi.com/2076-3417/10/24/9076/s1. Video S1-Dynamics of individual U87-MG cells in Collagen hydrogels. The movie shows U87-MG cells moving within the COL gel and assuming an elongated shape. In particular, following cell division (lower left quadrant; right side), U87-MG cells rapidly assume an elongated shape and move away from each other. Video S2-Dynamics 
of individual U87-MG cells in Hyaluronic Acid hydrogels. The movie shows moderate movement of rounded U87-MG cells with the HA gel. In particular, following cell division (upper right quadrant), U87-MG cells stay close to each other forming small clusters and preserving their original rounded shape. Video S3-Temporal evolution of U87-MG spheroids in Collagen hydrogels. The movie shows rapid development of the tumor spheroid with elongation and migration of U87-MG cells away from the original core towards the infiltrating region. An entire portion of the malignant mass tends to move away from the original core. Video S4-Temporal evolution of U87-MG spheroids in Hyaluronic Acid hydrogels. The movie shows a local development of the tumor spheroid with slow rearrangements in shape. No infiltrating region can be detected.

Author Contributions: Conceptualization, R.C.P., B.A.S. and P.D.; Data curation, R.C.P. and R.S.; Formal analysis, R.C.P., R.S. and D.P.B.; Funding acquisition, P.D.; Investigation, R.C.P., R.S., B.A.S. and P.D.; Methodology, R.C.P., R.S. and L.C.; Project administration, R.C.P., B.A.S. and P.D.; Resources, P.D.; Software, R.C.P., R.S., L.C. and D.P.B.; Supervision, B.A.S. and P.D.; Validation, R.C.P., R.S., B.A.S. and P.D.; Visualization, R.C.P., R.S., B.A.S. and P.D.; Writing-original draft, R.C.P. and P.D.; Writing-review \& editing, R.C.P., R.S., D.P.B., B.A.S. and P.D. All authors have read and agreed to the published version of the manuscript.

Funding: Research reported in this publication was supported by the National Cancer Institute of the National Institutes of Health under Award Number U54CA210181. The content is solely the responsibility of the authors and does not necessarily represent the official views of the National Institutes of Health. This project was further supported by the European Research Council, under the European Union's Seventh Framework Programme (FP7/2007-2013)/ERC grant agreement no. 616695, the AIRC (Italian Association for Cancer Research) under the individual investigator grant no. 17664 and by the Technical University of Munich-Institute for Advanced Study, funded by the German Excellence Initiative and the TÜV SÜD Foundation. D.P. Boso gratefully acknowledges the support from national funding under Grant DOR1718128.

Acknowledgments: The authors acknowledge the precious support provided by the Nikon Center at the Italian Institute of Technology for all microscopy acquisitions and analyses.

Conflicts of Interest: The authors declare no conflict of interest.

\section{Appendix A Numerical Solution and Computational Procedure}

The governing equations are discretized in space and time using a finite element method in space and a finite difference method in time, adopting a quasi-Crank-Nicolson scheme ( $\theta$ Wilson method with $\theta=0.52)[30,31]$. The weak form of the equations is obtained by means of the standard Galerkin procedure. Within each time step the equations are linearized by means of the Newton-Raphson method. The full set of equations is implemented by using Cast3M software, available at: http: $/ / w w w-c a s t 3 m . c e a . f r /$.

For the solution of the resulting system of equations, Equation (A1), a staggered scheme is adopted with iterations within each time step to preserve the coupled nature of the system.

$$
\mathbf{C}_{i j}(\mathbf{x}) \frac{\partial \mathbf{x}}{\partial t}+\mathbf{K}_{i j}(\mathbf{x}) \mathbf{x}=\mathbf{f}_{i}(\mathbf{x})
$$

with

$$
\begin{aligned}
\mathbf{C}_{i j} & =\left(\begin{array}{ccccccc}
\mathbf{C}_{n n} & 0 & 0 & 0 & 0 & 0 & 0 \\
0 & \mathbf{C}_{T T} & 0 & 0 & 0 & 0 & 0 \\
0 & 0 & \mathbf{C}_{E E} & 0 & 0 & 0 & 0 \\
0 & 0 & 0 & \mathbf{C}_{t t} & \mathbf{C}_{t h} & \mathbf{C}_{t l} & 0 \\
0 & 0 & 0 & \mathbf{C}_{h t} & \mathbf{C}_{h h} & \mathbf{C}_{t l} & 0 \\
0 & 0 & 0 & \mathbf{C}_{l t} & \mathbf{C}_{l h} & \mathbf{C}_{l l} & 0 \\
0 & 0 & 0 & 0 & 0 & 0 & \mathbf{C}_{u u}
\end{array}\right) \\
\mathbf{K}_{i j} & =\left(\begin{array}{ccccccc}
\mathbf{K}_{n n} & 0 & 0 & 0 & 0 & 0 & 0 \\
0 & \mathbf{K}_{T T} & 0 & 0 & 0 & 0 & 0 \\
0 & 0 & \mathbf{K}_{E E} & 0 & 0 & 0 & 0 \\
0 & 0 & 0 & \mathbf{K}_{t t} & \mathbf{K}_{t h} & \mathbf{K}_{t l} & 0 \\
0 & 0 & 0 & \mathbf{K}_{h t} & \mathbf{K}_{h h} & \mathbf{K}_{h l} & 0 \\
0 & 0 & 0 & \mathbf{K}_{l t} & \mathbf{K}_{l h} & \mathbf{K}_{l l} & 0 \\
0 & 0 & 0 & 0 & 0 & 0 & 0
\end{array}\right), \quad \mathbf{f}_{i}=\left(\begin{array}{c}
\mathbf{f}_{n} \\
\mathbf{f}_{T} \\
\mathbf{f}_{E} \\
\mathbf{f}_{t} \\
\mathbf{f}_{h} \\
\mathbf{f}_{l} \\
\mathbf{f}_{u}
\end{array}\right)
\end{aligned}
$$





in Sciumè et al. [31]. Three computational units are used in the staggered scheme: the first is for the nutrient mass fraction, the second to compute, $p^{h l}, p^{\text {th }}$, and $p^{l}$, and the third is used to obtain the displacement vector. Within each coupling iteration, the mass balance equation for the nutrient is solved for the mass fraction of the nutrient. Then the other mass balance equations are solved in a fully coupled way for $p^{h l}, p^{t h}$, and $p^{l}$, yielding here, de facto, $p^{t l}$ and $p^{l}$. In this second computational unit, at each iteration $i$ the approximate solution $p^{h l}, p^{\text {th }}$, and $p^{l}$ is used to update the mass fraction of the necrotic tumor cells, the mass exchange terms, and the reaction terms. Once convergence is achieved for the second computational unit, the pressure in the cells phases is used to compute the solid pressure. The solid pressure is needed to solve the momentum balance equations. Once convergence is achieved within a time step the procedure can march forward. It is interesting to note that the general procedure allows also for computations with one degree of saturation equal to zero without additional changes.

The modular computational structure allows to take into account more than one chemical species, simply adding a computational unit (equivalent to the first one used for the nutrient) for each of the additional chemical species considered. The procedure has been implemented in the code CAST3M (http://www-cast3m.cea.fr) of the French Atomic Energy Commission.

The input parameters for the model are the domain geometry and values of the physical governing parameters, which are listed in Tables 1-3. As output, the model yields the spatiotemporal evolution of each individual phase and tumor growth over time.

\section{References}

1. Louis, D.N.; Perry, A.; Reifenberger, G.; Von Deimling, A.; Figarella-Branger, D.; Cavenee, W.K.; Ohgaki, H.; Wiestler, O.D.; Kleihues, P.; Ellison, D.W. The 2016 World Health Organization classification of tumors of the central nervous system: A summary. Acta Neuropathol. 2016, 131, 803-820. [CrossRef] [PubMed]

2. Stupp, R.; Mason, W.P.; van den Bent, M.J.; Weller, M.; Fisher, B.; Taphoorn, M.J.B.; Belanger, K.; Brandes, A.A.; Marosi, C.; Bogdahn, U.; et al. Radiotherapy plus concomitant and adjuvant temozolomide for glioblastoma. N. Engl. J. Med. 2005, 352, 987-996. [CrossRef] [PubMed]

3. Cuddapah, V.A.; Robel, S.; Watkins, S.; Sontheimer, H. A neurocentric perspective on glioma invasion. Nat. Rev. Neurosci. 2014, 15, 455-465. [CrossRef] [PubMed]

4. Chen, H.-C. Boyden chamber assay. Cell Migr. 2005, 294, 15-22. [CrossRef]

5. Pampaloni, F.; Reynaud, E.G.; Stelzer, E.H.K. The third dimension bridges the gap between cell culture and live tissue. Nat. Rev. Mol. Cell Biol. 2007, 8, 839-845. [CrossRef]

6. Fraley, S.I.; Feng, Y.; Krishnamurthy, R.; Kim, D.-H.; Celedon, A.; Longmore, G.D.; Wirtz, D. A distinctive role for focal adhesion proteins in three-dimensional cell motility. Nat. Cell Biol. 2010, 12, 598-604. [CrossRef]

7. Taddei, M.L.; Giannoni, E.; Comito, G.; Chiarugi, P. Microenvironment and tumor cell plasticity: An easy way out. Cancer Lett. 2013, 341, 80-96. [CrossRef]

8. Venning, F.A.; Wullkopf, L.; Erler, J.T. Targeting ECM disrupts cancer progression. Front. Oncol. 2015, 5, 224. [CrossRef]

9. Murphy, A.R.; Laslett, A.; O'Brien, C.M.; Cameron, N.R. Scaffolds for 3D in vitro culture of neural lineage cells. Acta Biomater. 2017, 54, 1-20. [CrossRef]

10. Eccles, S.A.; Box, C.; Court, W. Cell migration/invasion assays and their application in cancer drug discovery. Biotechnol. Anпu. Rev. 2005, 11, 391-421. [CrossRef]

11. Valster, A.; Tran, N.L.; Nakada, M.; Berens, M.E.; Chan, A.Y.; Symons, M.H. Cell migration and invasion assays. Methods 2005, 37, 208-215. [CrossRef] [PubMed]

12. Rahman, A.; Carey, S.P.; Kraning-Rush, C.M.; Goldblatt, Z.E.; Bordeleau, F.; Lampi, M.C.; Lin, D.Y.; Garcia, A.J.; Reinhart-King, C.A. Vinculin regulates directionality and cell polarity in 2D, 3D matrix and 3D microtrack migration. Mol. Biol. Cell. 2016, 27, 1431-1441. [CrossRef] [PubMed]

13. Nikkhah, M.; Edalat, F.; Manoucheri, S.; Khademhosseini, A. Engineering microscale topographies to control the cell-substrate interface. Biomaterials 2012, 33, 5230-5246. [CrossRef] [PubMed] 
14. Rao, S.S.; DeJesus, J.; Short, A.R.; Otero, J.J.; Sarkar, A.; Winter, J.O. Glioblastoma behaviors in three-dimensional collagen-hyaluronan composite hydrogels. ACS Appl. Mater. Interfaces 2013, 5, 9276-9284. [CrossRef]

15. Pedron, S.; Becka, E.; Harley, B.A. Regulation of glioma cell phenotype in 3D matrices by hyaluronic acid. Biomaterials 2013, 34, 7408-7417. [CrossRef]

16. Wang, C.; Tong, X.; Yang, F. Bioengineered 3D brain tumor model to elucidate the effects of matrix stiffness on glioblastoma cell behavior using PEG-based hydrogels. Mol. Pharm. 2014, 11, 2115-2125. [CrossRef]

17. Yang, Y.-L.; Motte, S.; Kaufman, L.J. Pore size variable type I collagen gels and their interaction with glioma cells. Biomaterials 2010, 31, 5678-5688. [CrossRef]

18. Wang, C.; Tong, X.; Jiang, X.; Yang, F. Effect of matrix metalloproteinase-mediated matrix degradation on glioblastoma cell behavior in 3D PEG-based hydrogels. J. Biomed. Mater. Res. Part A 2017, 105, 770-778. [CrossRef]

19. Florczyk, S.J.; Wang, K.; Jana, S.; Wood, D.L.; Sytsma, S.K.; Sham, J.G.; Kievit, F.M.; Zhang, M. Porous chitosan-hyaluronic acid scaffolds as a mimic of glioblastoma microenvironment ECM. Biomaterials 2013, 34, 10143-10150. [CrossRef]

20. Koike, C.; McKee, T.D.; Pluen, A.; Ramanujan, S.; Burton, K.; Munn, L.L.; Boucher, Y.; Jain, R.K. Solid stress facilitates spheroid formation: Potential involvement of hyaluronan. Br. J. Cancer 2002, 86, 947-953. [CrossRef]

21. Santagiuliana, R.; Pereira, R.C.; Schrefler, B.A.; Decuzzi, P. Predicting the role of microstructural and biomechanical cues in tumor growth and spreading. Int. J. Numer. Methods Biomed. Eng. 2017, 34, e2935. [CrossRef] [PubMed]

22. Mascheroni, P.; Boso, D.; Preziosi, L.; Schrefler, B.A. Evaluating the influence of mechanical stress on anticancer treatments through a multiphase porous media model. J. Theor. Biol. 2017, 421, 179-188. [CrossRef] [PubMed]

23. Mascheroni, P.; Stigliano, C.; Carfagna, M.; Boso, D.P.; Preziosi, L.; Decuzzi, P.; Schrefler, B.A. Predicting the growth of glioblastoma multiforme spheroids using a multiphase porous media model. Biomech. Model. Mechanobiol. 2016, 15, 1215-1228. [CrossRef] [PubMed]

24. Hatzikirou, H.; Basanta, D.; Simon, M.; Schaller, K.; Deutsch, A. 'Go or grow': The key to the emergence of invasion in tumour progression? Math. Med. Biol. 2010, 29, 49-65. [CrossRef]

25. Giese, A.; Loo, M.A.; Tran, D.; Haskett, S.W. Coons BME Dichotomy of astrocytoma migration and proliferation. Int. J. Cancer 1996, 67, 275-282. [CrossRef]

26. Michor, F.; Liphardt, J.; Ferrari, M.; Widom, J. What does physics have to do with cancer? Nat. Rev. Cancer 2011, 11, 657-670. [CrossRef] [PubMed]

27. Moore, N.M.; Kuhn, N.Z.; Hanlon, S.E.; Lee, J.S.; Nagahara, L.A. De-convoluting cancer's complexity: Using a 'physical sciences lens' to provide a different (clearer) perspective of cancer. Phys. Biol. 2011, 8, 010302. [CrossRef]

28. Manneschi, C.; Pereira, R.C.; Marinaro, G.; Bosca, A.; Francardi, M.; Decuzzi, P. A microfluidic platform with permeable walls for the analysis of vascular and extravascular mass transport. Microfluid. Nanofluidics 2016, 20. [CrossRef]

29. Pereira, R.C.; Scaranari, M.; Castagnola, P.; Grandizio, M.; Azevedo, H.S.; Reis, R.L.; Cancedda, R.; Gentili, C. Novel injectable gel (system) as a vehicle for human articular chondrocytes in cartilage tissue regeneration. J. Tissue Eng. Regen. Med. 2009, 3, 97-106. [CrossRef]

30. Sciumè, G.; Shelton, S.; Gray, W.G.; Miller, C.T.; Hussain, F.; Ferrari, M.; Decuzzi, P.; Schrefler, B.A. A multiphase model for three-dimensional tumor growth. N. J. Phys. 2013, 15, 015005. [CrossRef]

31. Sciumè, G.; Santagiuliana, R.; Ferrari, M.; Decuzzi, P.; Schrefler, B.A. A tumor growth model with deformable ECM. Phys. Biol. 2014, 11, 065004. [CrossRef] [PubMed]

32. Gray, W.G.; Miller, C.T. Introduction to the Thermodynamically Constrained Averaging Theory for Porous Medium Systems, 1st ed.; Springer International Publishing: Berlin/Heidelberg, Germany, 2014; ISBN 978-3-319-04010-3.

33. Hawkins-Daarud, A.; Prudhomme, S.; Van Der Zee, K.G.; Oden, J.T. Bayesian calibration, validation, and uncertainty quantification of diffuse interface models of tumor growth. J. Math. Biol. 2012, 67, 1457-1485. [CrossRef] [PubMed]

34. Rocha, H.L.; Almeida, R.C.; Lima, E.A.B.F.; Resende, A.C.M.; Oden, J.T.; Yankeelov, T.E. A hybrid three-scale model of tumor growth. Math. Model. Methods Appl. Sci. 2017, 28, 61-93. [CrossRef] [PubMed] 
35. Lima, E.; Oden, J.T.; Hormuth, D.A., 2nd; Yankeelov, T.E.; Almeida, R.C. Selection, calibration, and validation of models of tumor growth. Math Models Methods Appl. Sci. 2016, 26, 2341-2368. [CrossRef]

36. Karageorgiou, V.; Kaplan, D.L. Porosity of 3D biomaterial scaffolds and osteogenesis. Biomaterials 2005, 26, 5474-5491. [CrossRef]

37. Tang, L.; Van De Ven, A.L.; Guo, D.; Andasari, V.; Cristini, V.; Li, K.C.; Zhou, X. Computational modeling of 3D tumor growth and angiogenesis for chemotherapy evaluation. PLoS ONE 2014, 9, e83962. [CrossRef]

38. Grinnell, F. Fibroblast biology in three-dimensional collagen matrices. Trends Cell Biol. 2003, 13, $264-269$. [CrossRef]

39. Christiansen, D.L.; Huang, E.K.; Silver, F.H. Assembly of type I collagen: Fusion of fibril subunits and the influence of fibril diameter on mechanical properties. Matrix Biol. 2000, 19, 409-420. [CrossRef]

40. Giese, A.; Kuwe, L.; Laube, B.; Berens, M.E. Migration of human glioma cells on myelin. Neurosurgery 1996, 38, 755-764. [CrossRef]

41. Toole, B.P. Hyaluronan: From extracellular glue to pericellular cue. Nat. Rev. Cancer 2004, 4, 528-539. [CrossRef]

42. Ananthanarayanan, B.; Kim, Y.; Kumar, S. Elucidating the mechanobiology of malignant brain tumors using a brain matrix-mimetic hyaluronic acid hydrogel platform. Biomaterials 2011, 32, 7913-7923. [CrossRef] [PubMed]

43. Soza, G.; Grosso, R.; Nimsky, C.; Hastreiter, P.; Fahlbusch, R.; Greiner, G. Determination of the elasticity parameters of brain tissue with combined simulation and registration. Int. J. Med. Robot 2005, 1, 87-95. [CrossRef] [PubMed]

44. Chatelin, S.; Constantinesco, A.; Willinger, R. Fifty years of brain tissue mechanical testing: From in vitro to in vivo investigations. Biorheology 2010, 47, 255-276. [CrossRef] [PubMed]

45. Cheng, S.; Clarke, E.C.; Bilston, L.E. Rheological properties of the tissues of the central nervous system: A review. Med. Eng. Phys. 2008, 30, 1318-1337. [CrossRef]

46. Kaufman, L.J.; Brangwynne, C.P.; Kasza, K.E.; Filippidi, E.; Gordon, V.D.; Deisboeck, T.S.; Weitz, D.A. Glioma expansion in collagen I matrices: Analyzing collagen concentration-dependent growth and motility patterns. Biophys. J. 2005, 89, 635-650. [CrossRef]

47. Ulrich, T.A.; Pardo, E.M.D.J.; Kumar, S. The mechanical rigidity of the extracellular matrix regulates the structure, motility, and proliferation of glioma cells. Cancer Res. 2009, 69, 4167-4174. [CrossRef]

48. Polyak, K.; Weinberg, R.A. Transitions between epithelial and mesenchymal states: Acquisition of malignant and stem cell traits. Nat. Rev. Cancer 2009, 9, 265-273. [CrossRef]

49. Guillamo, J.; Lisovoski, F.; Christov, C.; Le Guérinel, C.; Defer, G.; Peschanski, M.; Lefrançois, T. Migration pathways of human glioblastoma cells xenografted into the immunosuppressed rat brain. J. Neuro-Oncol. 2001, 52, 205-215. [CrossRef]

50. Kallenberg, K.; Goldmann, T.; Menke, J.; Strik, H.; Bock, H.C.; Stockhammer, F.; Buhk, J.H.; Frahm, J.; Dechent, P.; Knauth, M. Glioma infiltration of the corpus callosum: Early signs detected by DTI. J. Neuro-Oncol. 2013, 112, 217-222. [CrossRef]

51. Gritsenko, P.G.; Ilina, O.; Friedl, P. Interstitial guidance of cancer invasion. J. Pathol. 2012, 226, $185-199$. [CrossRef]

52. Marusyk, A.; Almendro, V.; Polyak, K. Intra-tumor heterogeneity: A looking glass for cancer? Nat. Rev. Cancer 2012, 12, 323-334. [CrossRef] [PubMed]

53. Brock, A.; Chang, H.; Huang, S. Non-genetic heterogeneity-A mutation-independent driving force for the somatic evolution of tumors. Nat. Rev. Genet. 2009, 10, 336-342. [CrossRef] [PubMed]

Publisher's Note: MDPI stays neutral with regard to jurisdictional claims in published maps and institutional affiliations. 